\title{
Finite temperature properties and frustrated ferromagnetism in a square lattice Heisenberg model
}

\author{
Nic Shannon ${ }^{1}$, Burkhard Schmidt ${ }^{2}$, Karlo Penc $^{3}$, and Peter Thalmeier ${ }^{2}$ \\ 1 SPEC, CEA Saclay, Orme des Merisiers F-91191 Gif sur Yvette CEDEX, France. \\ 2 Max-Planck-Institut für Chemische Physik fester Stoffe, Nöthnitzer Str. 40, 01187 Dresden, Germany. \\ 3 Research Institute of Solid State Physics and Optics, H-1525 Budapest, P.O.B. 49, Hungary \\ Received: date / Revised version: date
}

\begin{abstract}
The spin 1/2 Heisenberg model on a square lattice with antiferromagnetic nearest- and next-nearest neighbour interactions (the $J_{1}-J_{2}$ model) has long been studied as a paradigm of a two-dimensional frustrated quantum magnet. Only very recently, however, have the first experimental realisations of such systems been synthesized. The newest material, $\mathrm{Pb}_{2} \mathrm{VO}\left(\mathrm{PO}_{4}\right)_{2}$ seems to have mixed ferro- and antiferromagnetic exchange couplings. In the light of this, we extend the semiclassical treatment of the $J_{1}-J_{2}$ model to include ferromagnetic interactions, and present an analysis of the finite temperature properties of the model based on the exact diagonalization of 8,16 and 20 site clusters. We propose that diffuse neutron scattering can be used to resolve the ambiguity inherent in determining the ratio and sign of $J_{1}$ and $J_{2}$ from thermodynamic properties alone, and use a finite temperature Lanczos algorithm to make predictions for the relevant high temperature spin-spin correlation functions. The possibility of a spin-liquid phase occurring for ferromagnetic $J_{1}$ is also briefly discussed.
\end{abstract}

PACS. 71.27.+a Strongly correlated electron systems; heavy fermions - 71.10.-w Theory and models of manyelectron systems - 75.40.Cx Static properties (order parameter, static susceptibility, heat capacities, critical exponents, etc.)

\section{Introduction}

The antiferromagnetic (AF) Heisenberg model on a square lattice with added nearest neighbour bonds, commonly referred to as the $J_{1}-J_{2}$ model, has long served as a paradigm for a two dimensional frustrated AF. For a model with only one adjustable parameter - the ratio of next-nearest to nearest neighbour exchange $J_{2} / J_{1}$ - it has an extremely rich phase diagram, offering the chance to study Néel order (NAF) with a reduced sublattice magnetization for $J_{2} / J_{1} \ll 1$, a collinear AF (CAF) phase selected by an order from disorder effect for $J_{2} / J_{1} \gg 1$, and a spin-gapped phase (or family of phases) for intermediate coupling. A correspondingly rich and occasionally controversial literature, has accompanied the development of these ideas [1].

More recently, the discovery of high $\mathrm{T}_{c}$ superconductivity in doped layered cuprates whose undoped parent compounds are spin-half antiferromagnets, has lead to a renaissance of interest in 2D frustrated magnets. The $J_{1}-J_{2}$ model in particular has attracted renewed attention, both because of its simplicity and the possibility that its spin-gapped phase might provide a realization of Anderson's resonating valence bond (RVB) concept (see e.g. [2]).

Given all of this theoretical activity, it is surprising that the behaviour of the $J_{1}-J_{2}$ model in the presence of ferromagnetic (FM) exchange remains largely unexplored.

Frustrated FM's have an interesting history in their own right. The solid phases of He III have very complex magnetic behaviour determined by competing FM and AF multiple spin exchange processes. Under appropriate conditions He III may exhibit FM, AF or spin liquid ground states. Another, solid state, example is provided by the doped colossal magnetoresistance (CMR) manganites, where the competition between kinetic energy driven FM and superexchange driven AF is widely believed to result in a phase separation into regions with different magnetic order. It is therefore worth asking whether the $J_{1}-J_{2}$ model with FM $J_{1}$ shows similarly exotic behaviour.

It is also perhaps a little surprising, considering the wide variety of magnetic materials now under study, that the first " $J_{1}-J_{2}$ compound", $\mathrm{Li}_{2} \mathrm{VOSiO}_{4}$, was discovered only very recently [3, 4, 5]. $\mathrm{Li}_{2} \mathrm{VOSiO}_{4}$ is an insulating Vanadium oxide, with spin $S=1 / 2 \mathrm{~V}^{4+}$ ions arranged in square lattice planes, at the centres of $\mathrm{VO}_{4}$ pyramids. These are linked by $\mathrm{SiO}_{4}$ tetrahedra, with $\mathrm{Li}$ ions occupying the space between the $\mathrm{V}-\mathrm{O}$ planes. Because of this relatively complex structure, the magnetic ions are well separated, with weak superexchange between nearest and next-nearest neighbour $\mathrm{V}$ ions mediated by more than one intermediate $\mathrm{O}$ ion. A small number of related materials have now been synthesized, and the preliminary analysis of one of these, $\mathrm{Pb}_{2} \mathrm{VO}\left(\mathrm{PO}_{4}\right)_{2}$ seems to offer evidence of mixed FM and AF exchange coupling [6]. However, a precise and unambiguous measurement of the exchange couplings $J_{1}$ and $J_{2}$ by, e.g., inelastic neutron scattering, has yet to be accomplished for any of these new compounds. 
Motivated by $\mathrm{Pb}_{2} \mathrm{VO}\left(\mathrm{PO}_{4}\right)_{2}$, in this paper we provide an overview of the ground state and finite temperature properties of the $J_{1}-J_{2}$ with mixed FM and AF couplings. We present a comprehensive semiclassical analysis of the three dominant ordered phases of the model - a uniform FM phase, and $q=$ $(\pi, \pi)$ Néel (NAF) and $q^{*}=(\pi, 0),(0, \pi)$ collinear (CAF) antiferromagnetic phases - together with an exact analytic diagonalization of an eight site cluster, and finite temperature Lanczos results for the heat capacity and magnetic susceptibility for 16 and 20 site clusters.

We argue that, in addition to the known spin liquid region for $J_{1}>0, J_{2} \sim J_{1} / 2$, where the NAF and CAF phases compete, a new spin liquid region may exist for $J_{1}<0, J_{2} \sim-J_{1} / 2$, where the FM and CAF phases compete. We also propose that, because of their low magnetic energy scales, diffuse neutron scattering at finite temperatures can provide a very useful source of information about the nature of the competing magnetic interactions in these materials. With this in mind, we present the first quantitative numerical estimates of the magnetic structure factor $\mathrm{S}(q, \mathrm{~T})$ for the $J_{1}-J_{2}$ model.

\section{Zero temperature properties}

\subsection{Classical phase diagram and general arguments}

We consider the spin $1 / 2$ Heisenberg model on a square lattice

$$
\mathscr{H}=J_{1} \sum_{\langle i j\rangle_{1}} S_{i} \cdot S_{j}+J_{2} \sum_{\langle i k\rangle_{2}} S_{i} \cdot S_{k}
$$

where the sum on $\langle i j\rangle_{1}$ runs over nearest neighbour and the sum $\langle i k\rangle_{2}$ over diagonal next-nearest neighbour bonds. We allow the exchange constants $J_{1}$ and $J_{2}$ to be negative (FM) as well as positive (AF)

Since the properties of the $J_{1}-J_{2}$ model depend on the relative and not the absolute size of the exchange couplings $J_{1}$ and $J_{2}$, it is convenient to characterize it by an overall energy scale

$$
J_{\mathrm{c}}=\sqrt{J_{1}^{2}+J_{2}^{2}}
$$

and a frustration angle $\phi$ such that

$$
\begin{aligned}
J_{1} & =J_{\mathrm{c}} \cos (\phi) \quad J_{2}=J_{\mathrm{c}} \sin (\phi) \\
\phi & =\tan ^{-1}\left(J_{2} / J_{1}\right)
\end{aligned}
$$

As discussed in Section $3 J_{\mathrm{c}}$ can in principle be determined directly from the asymptotic behaviour of heat capacity and susceptibility at high temperatures. However the different physical properties of the model depend on the angle $\phi$, and this is much harder to determine from experiment.

Let us first consider the simplest possible classical analysis of the model. We assume that the system orders at zero temperature in such a way that all the spins are oriented in a common plane [7]. In this case the ground state energy of the $J_{1}-J_{2}$ model is minimised by an order parameter with wave vector $q=q^{*}$ such that the energy per spin

$$
E\left(q^{*}\right)=\frac{1}{2} z S^{2}\left[J_{1} \gamma_{1}\left(q^{*}\right)+J_{2} \gamma_{2}\left(q^{*}\right)\right]
$$

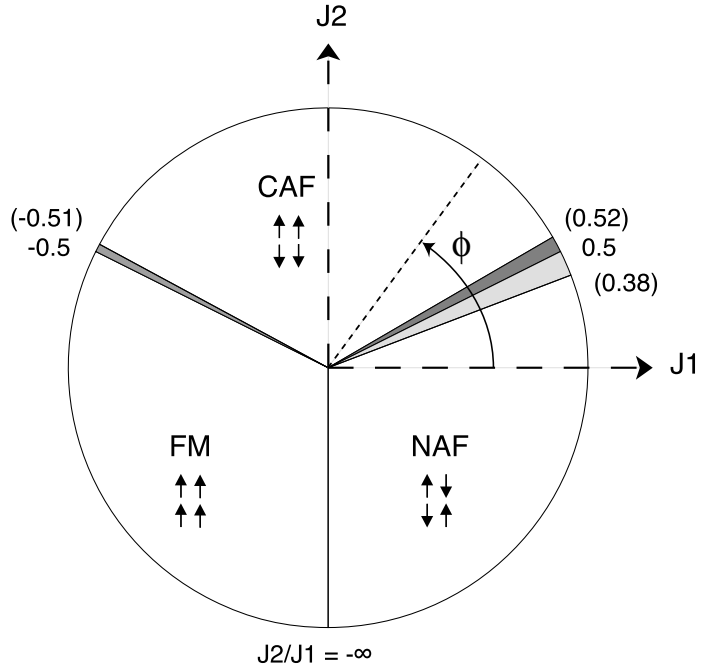

Fig. 1. Classical phase diagram. Numbers are ratios of exchange couplings $J_{2} / J_{1}$ for phase boundaries as determined from the classical ground state energy. The boundary between FM and NAF phase is the line $J_{1}=0, J_{2}<0\left(J_{2} / J_{1}=-\infty\right.$ in the figure). Values of $J_{2} / J_{1}$ in parentheses show where zero point fluctuations destroy the relevant order parameter at a semiclassical level, as discussed in Section 2.2 The shaded areas for $J_{1}>0$ correspond to the known spin-liquid regime, and for $J_{1}<0$ to another spin liquid region. The frustration angle is given by $\phi=\tan ^{-1}\left(J_{2} / J_{1}\right)$.

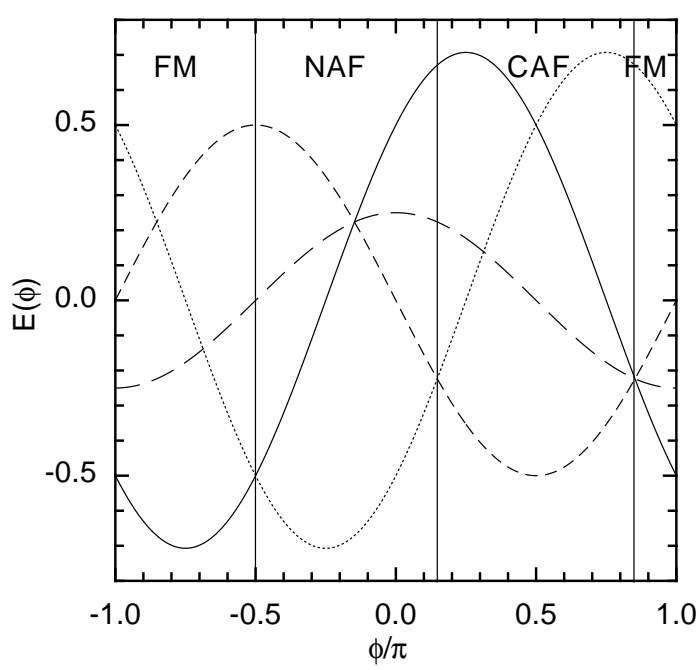

Fig. 2. Classical energies $E\left(q^{*}\right)$ as a function of the frustration angle $\phi$ in units of $J_{\mathrm{c}}$. Solid line: FM, $q^{*}=0$; dotted line: NAF, $q^{*}=(\pi, \pi)$; dashed line: CAF, $q^{*}=(\pi, 0)$; long-dashed line: four sublattice state for $q^{*}=(\pi / 2,0)$.

takes on its minimal value. Here

$$
\begin{aligned}
& \gamma_{1}(q)=\left(\cos \left(q_{x}\right)+\cos \left(q_{y}\right)\right) / 2 \\
& \gamma_{2}(q)=\cos \left(q_{x}\right) \cos \left(q_{y}\right)
\end{aligned}
$$

$z=4$ is the lattice coordination number for each type of bond and $S=1 / 2$ is the size of the spin. 


\begin{tabular}{lllll}
\hline & $q^{*}$ & Energy & Range $\left(J_{1}, J_{2}\right)$ & Range $\phi$ \\
\hline NAF & $(\pi, \pi)$ & $-J_{1} / 2+J_{2} / 2$ & $J_{1}>0, \quad J_{2}<J_{1} / 2$ & $-\pi / 2<\phi<\tan ^{-1}\left(\frac{1}{2}\right)$ \\
CAF & $(0, \pi)$ or $(\pi, 0)$ & $-J_{2} / 2$ & $\left|J_{2}\right|>\left|J_{1}\right| / 2$ & $\tan ^{-1}\left(\frac{1}{2}\right)<\phi<\pi-\tan ^{-1}\left(\frac{1}{2}\right)$ \\
FM & $(0,0)$ & $J_{1} / 2+J_{2} / 2$ & $J_{1}<0, \quad J_{2}<-J_{1} / 2$ & $\pi-\tan ^{-1}\left(\frac{1}{2}\right)<\phi<-\pi / 2$ \\
\hline
\end{tabular}

Table 1. Parameters for classical ground states diagram.

This analysis selects three phases, a Néel AF (NAF), a collinear $\mathrm{AF}(\mathrm{CAF})$ and a uniform FM to give the phase diagram shown in Figure 1 with the parameters given in Table 1 Note that the coplanar spiral states with $q^{*}=(2 \pi n / m, 0)$, where $\{n, m\}$ are integers, have energies which interpolate between the CAF and FM, and all of these states become degenerate exactly at the transition from FM to CAF. The classical energies of FM, NAF and CAF order parameters, together with a four sublattice state with $q^{*}=(\pi / 2,0)$ are shown in Figure 2

Each of the three lines selected as classical phase boundaries have interesting properties which are related to symmetries of the Hamiltonian. The mirror symmetry of the classical phase diagram about the line $J_{1}=0$ is particularly easy to understand. Since the square lattice is bipartite, the spins can be divided into $\mathrm{A}$ and $\mathrm{B}$ sublattices and, the Hamiltonian remains invariant under the transformation

$$
S^{B} \rightarrow-S^{B} \quad J_{1} \rightarrow-J_{1}
$$

This converts the classical NAF into a FM, and the classical $q^{*}=(0, \pi)$ CAF into the classical $q^{*}=(\pi, 0)$ CAF. Exactly on the line $J_{1}=0$ the A and B sublattices are entirely disconnected, so it is possible to rotate the classical NAF into the FM, and the classical $q^{*}=(\pi, 0)$ CAF into the classical $q^{*}=(0, \pi)$ $\mathrm{CAF}$, without any cost of energy.

Of course it is reasonable to ask whether such a naive classical picture has any relevance at all for the physics of a two dimensional frustrated spin 1/2 magnet. By way of an answer, let us consider in turn the simplest possible quantum analysis of the model.

Using the geometric trick of double counting all $J_{1}$ bonds and then setting $J_{1} \rightarrow J_{1} / 2$, the cross-linked square lattice can be expressed as four interpenetrating sublattices of edge sharing tetrahedra (cross-linked squares). And since each of these cross-linked squares is a complete graph, the Hamiltonian (1) can be rewritten in terms of the total spin on a square

$$
\mathscr{H}=-2 J_{2} S(S+1) N+\sum_{\square} \mathscr{H} \square
$$

where the sum runs over all $N$ squares $\square$ of the lattice, and

$$
\mathscr{H}_{\square}=\frac{J_{1}}{4} \Omega_{+}^{2}+\frac{1}{2}\left(J_{2}-\frac{J_{1}}{2}\right)\left[\Omega_{A}^{2}+\Omega_{B}^{2}\right]
$$

The spins within each square are counted clockwise (or counterclockwise) such that

$$
\begin{aligned}
\Omega_{+} & =\Omega_{A}+\Omega_{B} \\
\Omega_{A} & =S_{1}^{A}+S_{3}^{A} \\
\Omega_{B} & =S_{2}^{B}+S_{4}^{B}
\end{aligned}
$$

a)

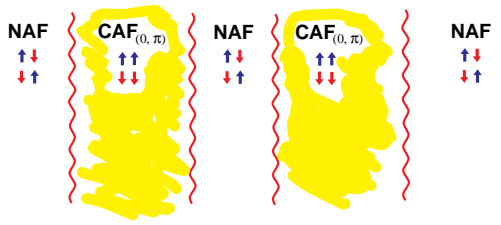

b)

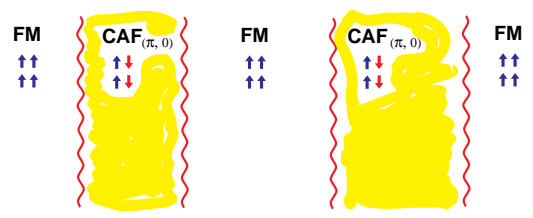

Fig. 3. On the transition lines a) $J_{2} / J_{1}=0.5$ and b) $J_{2} / J_{1}=-0.5$ the Ising domain wall energy between CAF and NAF or FM states vanishes and the system can break up into stripe like domains with all possible values of total magnetization.

With the Hamiltonian written in this way, the special role of the classical transition lines $J_{2} \pm J_{1} / 2=0$ becomes self-evident. Moreover, on these lines, the lowest energy is achieved by any state for which the sum (difference) of the A and B sublattice magnetisations vanishes in each square.

Classically, this condition can be satisfied by an exponentially large number of states. As a result, at these transitions, it becomes possible to break the system up into stripes. In the Ising limit these stripes are the alternating domains illustrated in Figure 3 The special (local) degeneracy of the Hamiltonian (7) also reveals itself in lines of zeros in the spinwave spectrum, discussed below.

We now use equation (7) to construct a minimal quantum theory for the model. The state of the whole system is determined if we specify all of the spins on any of the four sublattices of tetrahedra. So a lower quantum bound on the ground state energy can be obtained by considering a single isolated tetrahedron.

We find what are essentially the same three ground states as a function of $\phi$, with energies and quantum numbers given in Table 2 The full set of energy eigenvalues is shown in Figure 4 Apart from in the FM phase, where they must agree, the upper bound on the ground state energy obtained from the simple classical analysis, and the lower bound obtained from this minimal quantum estimate are generally quite different. However, it is far more significant that the same three phases are 
Nic Shannon et al.: Finite temperature properties and frustrated ferromagnetism in a square lattice Heisenberg model

\begin{tabular}{lllll}
\hline & $\left(\Omega, \omega_{A}, \omega_{B}\right)$ & Energy & Range $\left(J_{1}, J_{2}\right)$ & Range $\phi$ \\
\hline "NAF" & $(0,1,1)$ & $-J_{1}+J_{2} / 2$ & $J_{1}>0, \quad J_{2}<J_{1} / 2$ & $-\pi / 2<\phi<\tan ^{-1}\left(\frac{1}{2}\right)$ \\
"CAF" & $(0,0,0)$ & $-3 J_{2} / 2$ & $J_{2}>J_{1} / 2$ & $\tan ^{-1}\left(\frac{1}{2}\right)<\phi<\pi-\tan ^{-1}\left(\frac{1}{4}\right)$ \\
FM & $(2,1,1)$ & $J_{1} / 2+J_{2} / 2$ & $J_{1}<0, \quad J_{2}<-J_{1} / 4$ & $\pi-\tan ^{-1}\left(\frac{1}{4}\right)<\phi<-\pi / 2$ \\
\hline
\end{tabular}

Table 2. Parameters for the ground states of a single cross linked square.

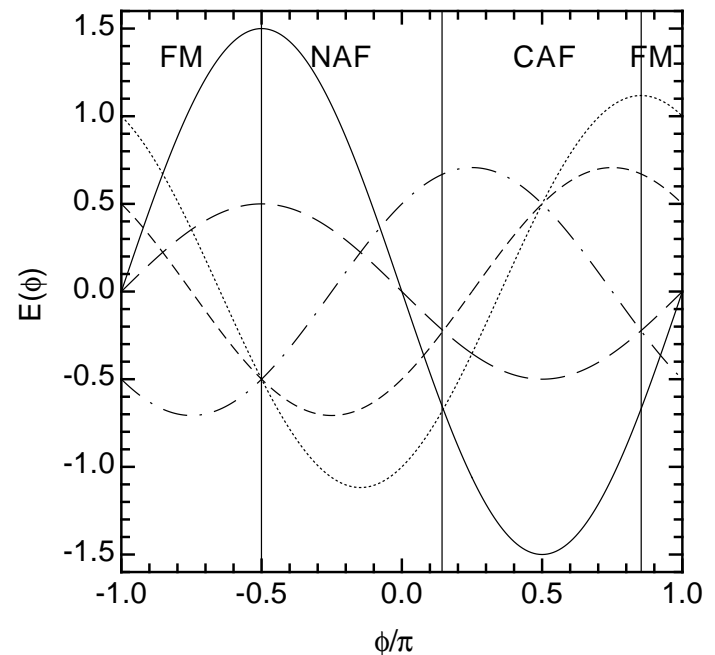

Fig. 4. Energies of the six eigenstates of a single tetrahedron, as a function of the frustration angle $\phi$, in units of $J_{\mathrm{c}}$.

found ${ }^{1}$, with the same phase boundaries - excepting that between the CAF and FM, where quantum fluctuations extend the CAF regime at the expense of the FM regime.

This simple correspondence between the most naive classical and quantum theories lends us some confidence in both. And although neither can give a complete description of the model, both can be improved systematically, as discussed in later sections of the paper. The key question which remains is how the phase transitions between the three dominant phases take place. The existence of an extensive set of spiral states degenerate with the FM and CAF order parameters at $\phi=$ $\pi-\tan ^{-1}(1 / 2)$, and the complicated level crossings for the tetrahedron near $\phi=\tan ^{-1}(1 / 2)$ and $\phi=\pi-\tan ^{-1}(1 / 2)$ already hint that these can be non-trivial.

\subsection{Semiclassical spin wave analysis}

The first step to improving on the naive classical phase diagram is to consider the influence of semiclassical spin wave excitations. While for the FM, spin waves are eigenstates, the frustrated NAF and CAF phases both show marked fluctuations at zero temperature. All three phases must be unstable at finite

\footnotetext{
1 Here "phase" should be understood to mean a ground state wave function with the same total spin and spatial symmetries as the corresponding classical order parameter - SU(2) invariance clearly cannot be spontaneously broken in a small cluster.
}

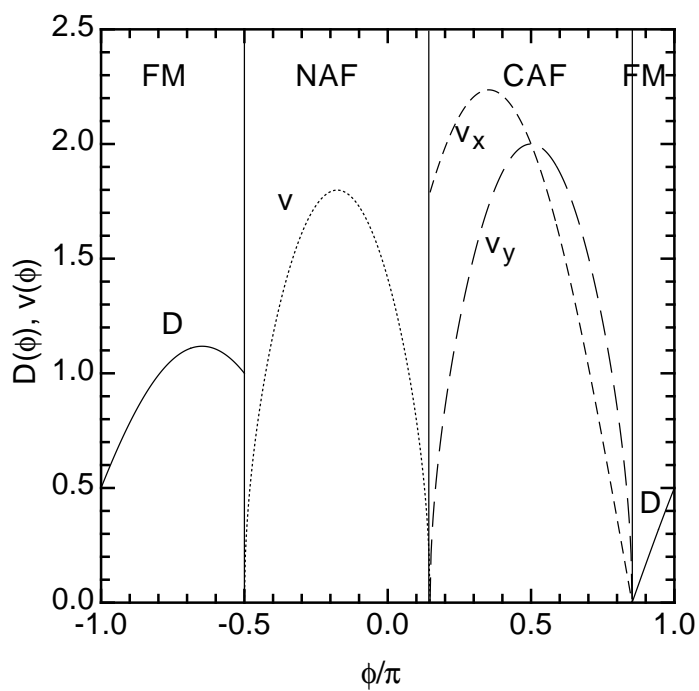

Fig. 5. Evolution of the spin stiffness $D$ in the FM phase (solid line), and of the spinwave velocities $v$ in the NAF phase (dotted line) and $v_{x}$, $v_{y}$ in the CAF phases (dashed lines), as a function of the frustration angle $\phi$ in units such that the lattice constant $a=1$ and $J_{\mathrm{c}}=1$.

temperatures, as long range order would violate the MerminWagner theorem. None the less, linear spin wave theory captures the essential physics of the ordered phases, and provides some interesting hints about how the classical phase diagram must be modified in the quantum case.

\subsubsection{FM phase}

Expanding about the FM phase we find a spin wave dispersion

$$
\omega(q)=-4 S\left[J_{1}+J_{2}\right]+4 S\left[J_{1}\left(c_{x}+c_{y}\right) / 2+J_{2} c_{x} c_{y}\right]
$$

where

$$
c_{x}=\cos \left(q_{x}\right) \quad c_{y}=\cos \left(q_{y}\right)
$$

in units such that the lattice constant $a=1$. The spin wave dispersion for a range of values of $\phi$ throughout the FM phase is shown in Figure 6 Note that in this and all subsequent plots of spin wave dispersion, the $q_{x}$ and $q_{y}$ values run from $-\pi$ to $\pi$, i. e. over the full Brillouin zone (BZ) for the square lattice, and not the reduced magnetic BZ's appropriate to the NAF or CAF phases.

While the fully polarized FM ground state remains an exact eigenstate of the frustrated model, its dispersion is profoundly modified by competing interactions. At the boundary with the 

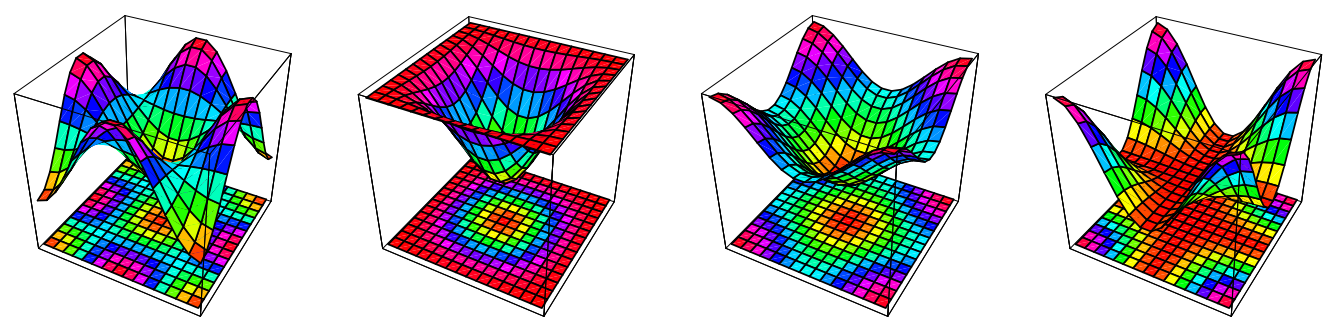

Fig. 6. Evolution of spinwave dispersion in FM phase. From left to right - border with NAF, deep within FM phase, pure nearest neighbour exchange, border with CAF.

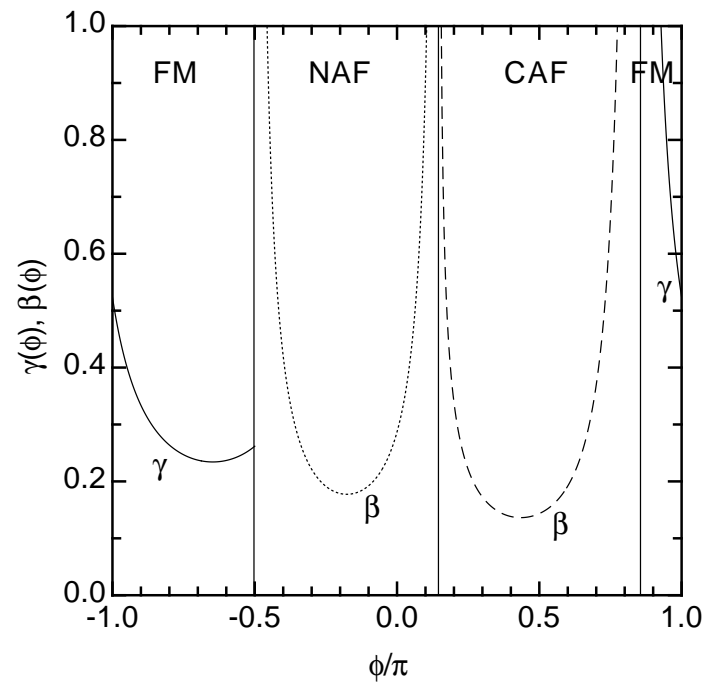

Fig. 7. Evolution of the heat capacity $C_{V}$ as a function of the frustration angle $\phi$. In FM regions the quantity plotted is the prefactor $\gamma$ of $C_{V}=\gamma T$, and in AF regions the prefactor $\beta$ of $C_{V}=\beta T^{2}$, where temperature is measured in units of $J_{\mathrm{c}}$.

NAF phase for $\phi_{c}=-\pi / 2$, the dispersion is that of a pure $J_{2}$ FM, which has the same magnetic BZ as the NAF phase, and therefore zeroes at $q=(\pi, \pi)$ and symmetry points, in addition the usual $q=(0,0)$ Goldstone mode. Deep within the FM phase for $\phi=-\pi+\tan ^{-1}(1 / 2)$, the dispersion behaves as

$$
\omega(q) \sim D q^{2}
$$

where the stiffness constant $\mathrm{D}$ is given by

$$
D=-\left(J_{1}+2 J_{2}\right) S
$$

in the zone centre, but vanishes on the zone boundary. The variation of $D$ as a function of $\phi$ is plotted in Figure 5 For $\phi=-\pi$ the dispersion is that of the familiar pure $J_{1}$ FM. And, finally, on the boundary between FM and CAF for $\phi_{c}=\pi-\tan ^{-1}(1 / 2)$, the dispersion vanishes on the lines $q_{x}=0$ and $q_{y}=0$. These lines of zeros are a direct manifestation of the special local symmetry discussed in Section 2.1

The heat capacity of a FM in 2D is linear at low temperatures, reflecting a constant density of states at zero energy, and scales as

$$
C_{V}=\frac{\zeta(2)}{2 \pi}\left(\frac{T}{D}\right)
$$

where $\zeta(2)=\pi^{2} / 6$. The coefficient of $T$ as function of $\phi$ is plotted in Figure 7 It diverges at the transition between the FM and the CAF, but approaches a constant at the transition between FM and NAF.

\subsubsection{NAF phase}

The spinwave spectrum for the NAF is given by

$$
\omega(q)=\sqrt{A_{q}^{2}-B_{q}^{2}}
$$

where the coupling between spins on a given sublattice is

$$
A_{q}=4 S\left[J_{1}-J_{2}\left(1-c_{x} c_{y}\right)\right]
$$

and the coupling between the two sublattices is

$$
B_{q}=2 J_{1} S\left(c_{x}+c_{y}\right)
$$

Where $J_{2}$ is FM, it acts to stabilize the NAF order, where $J_{2}$ is $\mathrm{AF}$, it acts to destroy it. Once again this competition is visible in the spin wave dispersion, as show in Figure 8

At the boundary with the FM phase for $\phi_{c}=-\pi / 2$, the dispersion is that of a pure $J_{2} \mathrm{NAF}$, and exactly matches that of the FM on this phase boundary. Deep within the NAF phase for $\phi_{c}=-\tan ^{-1}(1 / 2)$, the low energy spin wave dispersion behaves as

$$
\omega(q) \sim v_{s}\left|q-q^{*}\right|
$$

where the isotropic spin wave velocity is given by

$$
v_{s}=2 S \sqrt{2 J_{1}\left(J_{1}-2 J_{2}\right)}
$$

and $q^{*}=(\pi, \pi)$, as expected. However it exhibits a marked dispersion about the magnetic zone boundary, as compared to the pure $J_{1}$ NAF for $\phi=0$. Finally, on the boundary with the CAF phase for $\phi_{c}=\tan ^{-1}(1 / 2)$, the dispersion vanishes on the lines $q_{x}=0, q_{x}= \pm \pi$ and $q_{y}=0, q_{y}= \pm \pi$. Values of the spinwave velocity $v_{S}$ are shown in Figure 5 in units in such that the lattice spacing $a=1$ and the overall energy scale $J_{\mathrm{c}}=1$. 

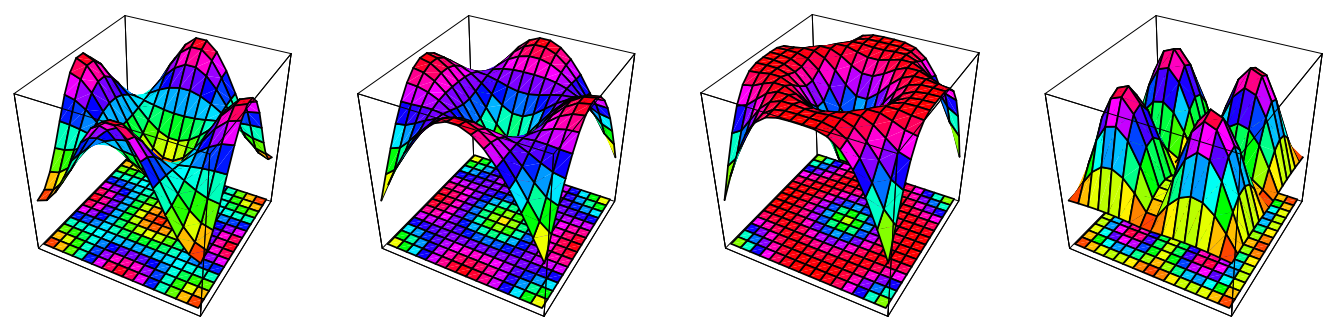

Fig. 8. Evolution of spinwave dispersion in NAF phase. From left to right - border with FM, deep within NAF phase, pure nearest neighbour exchange, border with CAF.

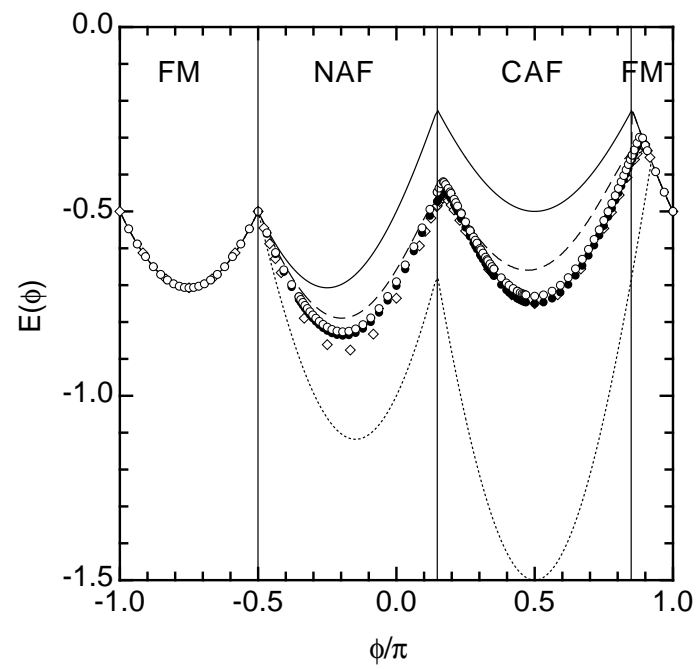

Fig. 9. Different estimates of the ground state energy per spin as a function of the frustration angle $\phi$ in units of $J_{\mathrm{c}}$. Uppermost (solid) line - classical energy of FM, NAF and CAF order parameters given by Equation 4 Lowermost (dotted) line - lower quantum bound given by the ground state of the Hamiltonian (7). Inner (dashed) line - semiclassical spinwave theory. Diamonds - variational lower bound based on diagonalization of a nine-site cluster. Solid and open circles ground state energy obtained from exact diagonalization of a 16- and 20-site cluster, respectively. Note that in the collinear phase the variational bound from the nine-site cluster and the exact-diagonalization result for the 16-site cluster nearly coincide.

The low temperature heat capacity of the NAF is controlled by the spin wave velocity and is given by

$$
C_{V}=\frac{3 \zeta(3)}{2 \pi}\left(\frac{T}{v_{S}}\right)^{2}
$$

where $\zeta(3)=1.202 \ldots$. The relevant coefficient of $T^{2}$, as a function of $\phi$ is plotted in Figure 7

The quantum zero point corrections to the ground state energy also vary strongly as a function of $\phi$ within the NAF state, vanishing altogether at the boundary with the FM. These are shown in Figure 9 together with the upper classical and lower quantum bounds discussed in Section 2.1. In fact, near the transition from NAF to CAF, the semiclassical estimate of the ground state energy surprisingly well with series expansion

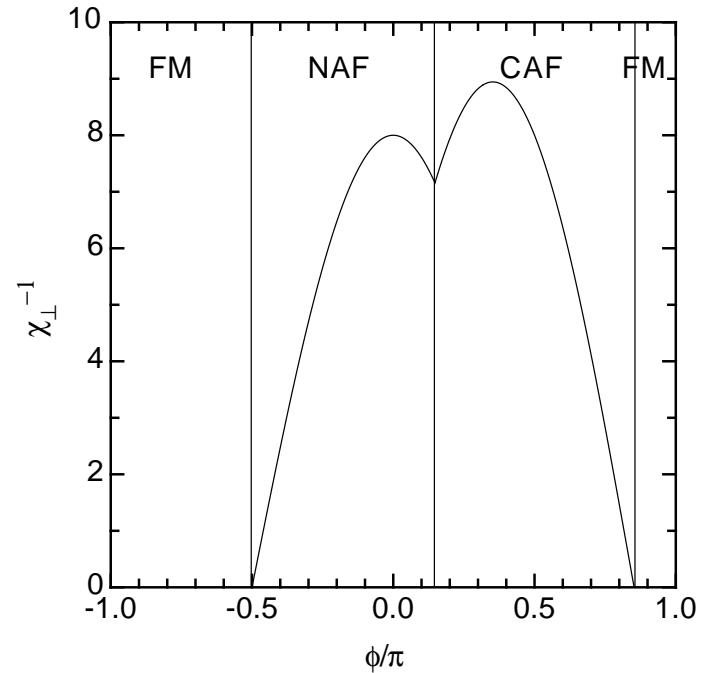

Fig. 10. Evolution of the inverse transverse susceptibility $\chi_{\perp}^{-1}$ as a function of the frustration angle $\phi$, in units such that $J_{\mathrm{c}}=1$ and $\left(g \mu_{\mathrm{B}}\right)^{2}=1$.

estimates [8]. It also lies very close to our numerical estimates and an improved variational quantum bound, discussed below. These are also plotted in Figure 9

It is also interesting to consider the $\phi$ dependence of the experimentally accessible transverse susceptibility $\chi_{\perp}$ which, within spin wave theory for a two sublattice $\mathrm{AF}$ is given by

$$
\chi_{\perp}^{-1}=\frac{2}{S} A_{q=0}
$$

in units such that the overall prefactor $\left(g \mu_{B}\right)^{2}=1$. The variation of the inverse susceptibility as a function of $\phi$, normalized to the value for $\phi=0$, is shown in Figure 10 The susceptibility is a continuous function of $\phi$, diverging (as $\chi_{\perp} \sim\left(\phi-\phi^{*}\right)^{-1}$ ) at the FM phase boundary, and matching that of the CAF at the other end of the NAF phase. However its derivative is discontinuous across each transition, reflecting the changing symmetry of the order parameter.

We can gain still more information about the evolution of the NAF state for different couplings by calculating the sublattice magnetisation $m_{S}$. Quantum fluctuations reduce $m_{S}$ from its classical value $M_{A}=1 / 2$, and in terms of the coefficients 


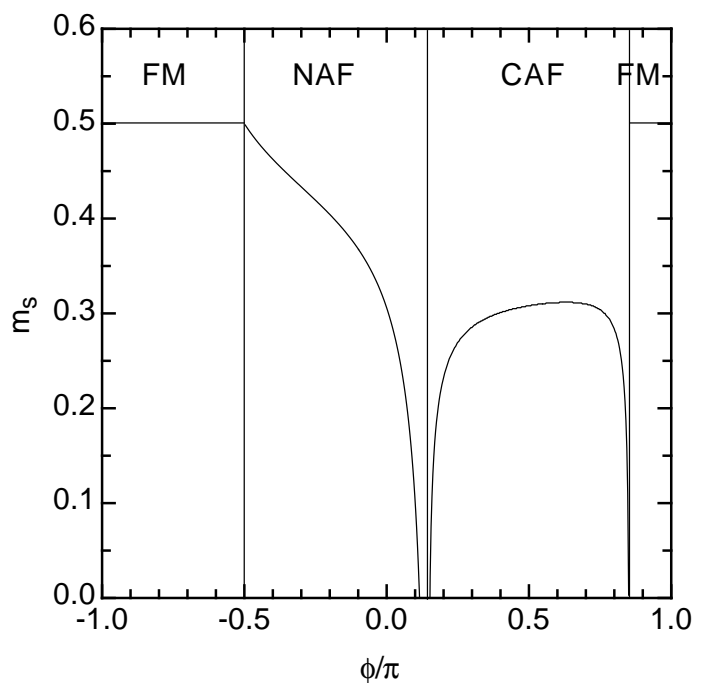

Fig. 11. Evolution of sublattice magnetization $m_{s}$ as a function of the frustration angle $\phi$.

defined above, it is given by:

$$
m_{S}=\frac{1}{2} \sum_{q}\left[1-\frac{A_{q}}{\sqrt{A_{q}^{2}-B_{q}^{2}}}\right]
$$

The variation of $m_{s}$ as a function of $\phi$ is shown in Figure 11 The sublattice magnetization of the NAF vanishes for $\phi / \pi=$ 0.12 , corresponding to a ratio of $J_{2} / J_{1}=0.38$. This result implies that, at a semiclassical level, the NAF order parameter is destroyed by fluctuations long before the competing CAF becomes energetically favourable. Historically, this was the first signature of the existence of an intermediate, spin-gapped phase between the NAF and CAF in the purely AF $J_{1}-J_{2}$ model [9]. We return to this point in the context of the model with mixed $\mathrm{FM}$ and $\mathrm{AF}$ couplings below.

\subsubsection{CAF Phase}

The CAF phase can be thought of as two interpenetrating NAF lattices of diagonal $J_{2}$ bonds. At a purely classical level, these sublattices are decoupled and can be rotated freely about one another. However quantum fluctuations stabilise the configuration in which the Néel vectors of both sublattices, and therefore the associated spins, are collinear. This tendency of fluctuations to favour collinear spin configurations is well known [10, 11, [12], and for small $J_{1} / J_{2}$ is independant of the sign of $J_{1}$. We have checked explicitly that this order from disorder effect survives for larger, FM values of $J_{1}$ by peforming spin wave calculations for more general four-sublattice states. Details of these will be reproduced elsewhere. We note that any further twosublattice canting of the CAF state is energeticaly unfavourable at a classical level, even for quite large FM $J_{1}$.

Once the two sublattice CAF has been selected, the analysis of the spinwave spectrum is straightforward. The spinwave spectrum for the CAF is once again of the form equation (17).
For the CAF order parameter with $q^{*}=(\pi, 0)$ we find

$$
\begin{aligned}
& A_{q}=2 S\left[2 J_{2}+J_{1} c_{y}\right] \\
& B_{q}=2 S c_{x}\left[J_{1}+2 J_{2} c_{y}\right]
\end{aligned}
$$

in accordance with [9] (the result for $q^{*}=(0, \pi)$ can be obtained simply by exchanging $x$ and $y$ above). The evolution of the spin wave dispersion within the CAF phase is shown in Figure 8 plotted within the full $\mathrm{BZ}$ for the square lattice.

At the border with the NAF phase, for $\phi_{c}=\tan ^{-1}(1 / 2)$ the CAF has lines of zero modes for $q_{x}=0, q_{x}= \pm \pi$ and $q_{y}= \pm \pi$, but not for $q_{y}=0$, and has maxima at $q=( \pm \pi / 2,0)$. Within the CAF phase for AF couplings, for $\phi=\pi / 4$, the dispersion has peaked maxima at $q=( \pm \pi / 2,0)$, dispersionless ridges for $q_{y}= \pm \pi / 2$. In all cases the dispersion had zeros at the wave vectors appropriate to the order parameter, i.e. $q=(0,0)$ and $q^{*}=( \pm \pi, 0)$. Near to these we find a linear but anisotropic spin wave dispersion

$$
\omega(q) \sim \sqrt{\left[v_{s}^{x}\left(q_{x}-q_{x}^{*}\right)\right]^{2}+\left[v_{s}^{y}\left(q_{y}-q_{y}^{*}\right)\right]^{2}}
$$

where the spin wave velocities are given by

$$
\begin{aligned}
v_{S}^{x} & =2 J_{2}+J_{1} \\
v_{S}^{y} & =\sqrt{\left(2 J_{2}+J_{1}\right)\left(2 J_{2}-J_{1}\right)}
\end{aligned}
$$

The low temperature heat capacity of the CAF is controlled by the average spin wave velocity $\tilde{v}_{S}=\left(v_{S}^{x} v_{S}^{y}\right)^{\frac{1}{2}}$ and is given by the equivalent of equation (16)

$$
C_{V}=\frac{3 \zeta(3)}{2 \pi}\left(\frac{T}{\tilde{v}_{S}}\right)^{2}
$$

where $\zeta(3)=1.202 \ldots$. The relevant coefficient of $T^{2}$, as a function of $\phi$ is plotted in Figure 7

The symmetry between the $\mathrm{x}$ and $\mathrm{y}$ axes is broken by the CAF order parameter, which is reflected in the different values of $v_{x}$ and $v_{y}$. However, at a semiclassical level, this symmetry breaking is not reflected in different values the transverse susceptibility $\chi_{\perp x}$ and $\chi_{\perp y}$ - plotted in Figure 10 This can be understood as follows - the transverse susceptibility associated with an AF can be expressed in terms of the spin stiffness $\rho_{s}$ and the spinwave velocity $v_{s}$ using the hydrodynamic relation $\chi_{\perp}=\rho_{s} / v_{s}^{2}$. However at this level of approximation the variation of $\mathrm{D}$ with angle in the plane is precisely that required to cancel the variation of $v_{s}$. Once again, the special role of the lines $J_{1}= \pm 2 J_{2}$ is evident - at the borders of the CAF phase the solutions for $v_{y}$ become imaginary, while the transverse susceptibility diverges.

For the pure next-nearest neighbour model at $\phi=\pi / 2$, the magnetic BZ is further reduced, with additional minima at $q=( \pm \pi, \pm \pi)$ and $q_{y}= \pm \pi$. The dispersion exhibits ridge like maxima for $q=( \pm \pi / 2, \pm \pi / 2)$. Within the CAF phase for partially FM coupling at $\phi=3 \pi / 4$, the dispersion is that for $\phi=\pi / 4$ described above, but with $q$ translated by $(\pi / 2,0)$. Finally, for $\phi_{c}=\pi-\tan ^{-1}(1 / 2)$, at the boundary with the FM state, it has maxima for $q=( \pm \pi / 2, \pm \pi)$ and line zeros for $q_{x}= \pm \pi$ and $q_{y}=0, q_{y}= \pm \pi$, but not for $q_{x}=0$.

While it was possible to exactly match up the spin wave dispersion on the boundary between the NAF and FM, where 
the A and B sublattices decouple, this cannot be done for the boundaries between the CAF and NAF or CAF and FM. The different way in which the CAF order parameter breaks the lattice symmetry is immediately apparent in the different number of lines of zero modes present in the CAF for $\phi=\tan ^{-1}(1 / 2)$ and $\phi=\pi-\tan ^{-1}(1 / 2)$, as compared with the NAF or FM.

This rules out a smooth transition from one state to the other, and at a semiclassical level, the only way in which the system can avoid embarrassment is to dissolve the classical CAF order parameter before the phase boundary is reached. And indeed the relevant sublattice magnetisation does vanish for $\phi / \pi=0.15\left(J_{2} / J_{1}=0.52\right)$, as the CAF approaches the $\mathrm{NAF}$, and for $\phi / \pi=0.85\left(J_{2} / J_{1}=-0.51\right)$, as the CAF approaches the FM phase. Consistent with this, the heat capacity (Figure 7) shows clear anomalies as a function of $\phi$ at either end of the CAF phase.

\subsection{Beyond the semiclassical picture}

The simple quantum and semiclassical arguments presented above give rise to an equally simple and self consistent picture of the phase diagram of the $J_{1}-J_{2}$ model as a function of $\phi$. The model has three dominant phases, FM, NAF and CAF. The phase transition between the FM and NAF is straightforward. The phase transitions between the CAF and NAF, and the CAF and FM are not, and probably, take place through an intermediate phase.

The different estimates of the ground state energy of the system in Figure 9 give us further reason to believe that this semiclassical picture is not far from the truth. Clearly the spin wave estimate of the ground state energy lies a long way above the lower bound from the tetrahedral cluster. But, as discussed in Appendix $C$ it is possible to construct a much better quantum bound variationally, from the numerical diagonalization of a 9 site clusters with modified boundary conditions. Increasing the cluster size to 13 sites does not lead to any significant change in the ground state energy. Since the semiclassical estimate is within a few percent of the quantum bound on the ground state energy for almost all values of $\phi$, there is little room for drastic changes in the phase diagram.

But of course this is not the end of the story. Even if our semiclassical estimate of zero point energy is reliable, fluctuations will strongly renormalise the semiclassical spin wave spectra and correlation functions. In the pure $J_{1}$ Néel AF [13] these corrections lead to an enhancement of the spin wave velocity

$$
v_{S} \rightarrow Z_{c} v_{S} \quad Z_{c}=1+\frac{0.1580}{2 S}+\frac{0.0216}{(2 S)^{2}}+\ldots \approx 1.1794
$$

a suppression of the perpendicular susceptibility

$$
\chi_{\perp} \rightarrow Z_{\chi} \chi_{\perp} \quad Z_{\chi}=1-\frac{0.551}{2 S}+\frac{0.065}{(2 S)^{2}}+\ldots \approx 0.514
$$

and a slight enhancement of the sublattice magnetisation,

$$
m_{S} \rightarrow Z_{m} S \quad Z_{m}=1-\frac{0.383}{2 S}+\frac{0.007}{(2 S)^{2}}+\ldots \approx 0.613
$$

The values of the coefficients $Z$ as a function of $J_{1}$ and $J_{2}$ for $J_{2} \neq 0$ remain to be calculated, and until they are known, quantitative comparison of the numbers found above with experiment must be approached with some caution. However experience with the pure $J_{1}$ model suggests that, as long as fluctuations do not destroy magnetic order completely, corrections are reasonably uniform and the semiclassical description of spin correlations remains qualitatively, if not quantitatively valid.

This "renormalised classical" physics should be expected to break down near the highly frustrated transitions into, and out of the CAF phase. The large body of existing work on the $J_{1}-J_{2}$ model with AF couplings suggests that the space between the NAF and CAF phases is filled by a spin-gapped 'spin-liquid' phase. Spin liquids are known to occur adjacent to a FM phase in the Heisenberg model on a triangular lattice with competing FM and AF cyclic exchanges [14], and on a honey-comb lattice with competing $J_{1}, J_{2}$ and $J_{3}$ (next-next nearest neighbour) interactions [15].

So what happens between the FM and CAF phases in our model ?

At least at first glance, the situation seems to be very similar. A high local degeneracy in the classical spectrum - a family of degenerate order parameters for $\phi=\pi-\tan ^{-1}(1 / 2)-$ leads to line zeros in the spin wave velocity and vanishing sublattice magnetisation exiting the CAF state towards the FM. Exactly the same things happen at the much studied boundary with the NAF. This similarity is by no means proof of the existence of a spin liquid state in the $J_{1}-J_{2}$ model with FM $J_{1}$, but it is a sufficient reason to start looking for one.

Further circumstantial evidence in favour of this hypothesis can be obtained from the exact analytic diagonalization of an 8-site cluster described in Appendix B The resulting energy spectrum, classified by spin, is shown in Figure 13 The straight-forward phase transition between NAF and FM states for $\phi=-\pi / 2$ shows up as a multiple crossings of ground state and excitation energy levels, all of which take place at the same critical value of $\phi=-\pi / 2$.

Where the singlets associated with NAF and CAF order parameters cross, the reordering of excited states does not take place at a single critical value of $\phi$, but is spread out over a finite range of $\phi$. Simply counting where the lowest lying triplet excitation crosses the lowest lying singlet excitation either side of the ground state crossing gives a remarkably good (if arbitrary) estimate of the extent of the spin liquid region - from $J_{1} / J_{2}=$ 0.38 to $J_{1} / J_{2}=0.60$, values which are comparable with those found in the existing literature [2, 5, 9, 16, 17, 18, 19, 20].

Examining the level crossings associated with the transition from CAF to FM we see the same extended structure. In this case applying the same naive criterion based on the crossing of first excitations would predict a spin liquid region from $J_{1} / J_{2}=-0.38$ to $J_{1} / J_{2}=-0.60$. However, in order to obtain a more serious numerical estimate of the domain of stability of the CAF and FM order parameters it would be necessary to look at the finite size scaling of not only the first excitation energies, but of the entire "Anderson Tower" of states [21, 22, 23] which go to make up the CAF order parameter in the thermodynamic limit, for a sequence of clusters including those of relatively large sizes (e.g. 32 and 36 sites). This analysis is beyond the scope of the present paper. 

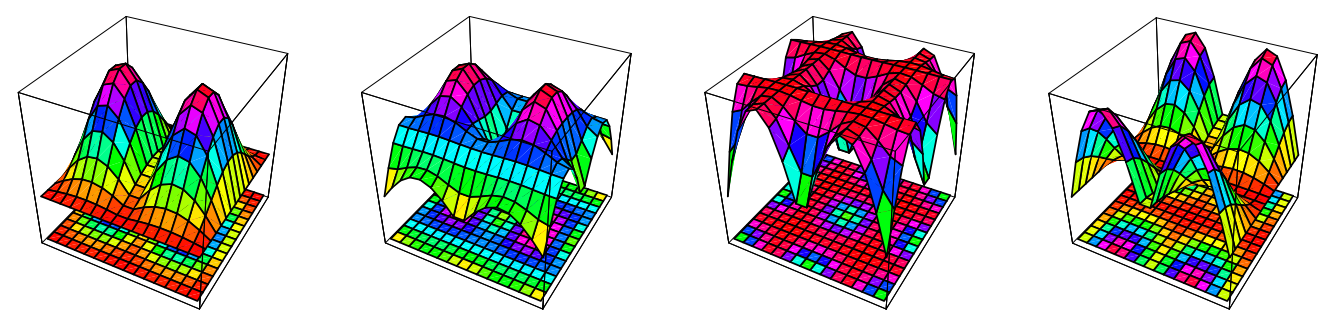

Fig. 12. Evolution of spinwave dispersion in CAF phase. From left to right - border with NAF, within CAF phase for AF couplings, pure next nearest neighbour exchange, border with FM.

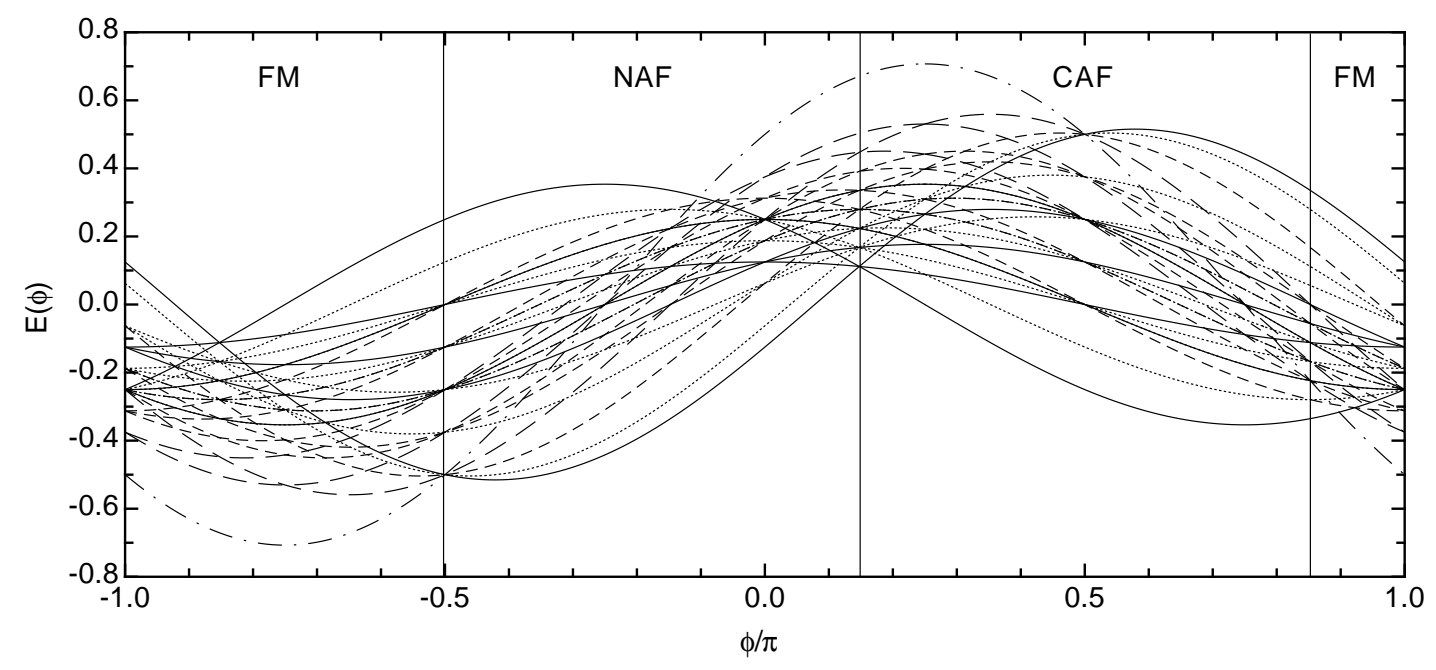

Fig. 13. Energy levels (per spin) of the 8 site cluster, classified according to total spin $\Omega$ as a function of the frustration angle $\phi$, in units of $J_{\mathrm{c}}$ : solid lines $-\Omega=0$ (singlet); dotted lines $-\Omega=1$ (triplet); short-dashed lines $-\Omega=2$; long-dashed lines $-\Omega=3$; dash-dotted line $-\Omega=4$ (maximal spin).

It also seems premature to speculate about the nature of any possible new phase appearing between the FM and CAF. Thinking classically, one might imagine that a spiral or canted state arises which interpolates between the CAF and FM. Adding an $\mathrm{AF} J_{3}$ interaction to the model would tend to favour such states (cf. [12]). However our preliminary analysis suggests that, for the relevant range of $J_{1}$ and $J_{2}$ (with $J_{3} \equiv 0$ ), these states are still more unstable against fluctuations than the CAF with which they compete.

In short, while the outcome remains uncertain, there is clearly reason to suspect that something interesting happens at the transition from CAF to FM. In marked contrast to the $J_{1}-J_{2}$ model with $\mathrm{AF}$ interactions, the existing literature on this problem appears to be in its infancy [24, 25].

\section{Finite temperature properties}

\subsection{General considerations}

The energy and temperature scales of the $J_{1}-J_{2}$ model are controlled by the single parameter $J_{\mathrm{c}}$. In principle, this can be determined directly from the asymptotic behaviour of the magnetic contribution to the heat capacity at high temperatures 18 ,
19, 26]

$$
C_{V}(T \rightarrow \infty)=\frac{3}{8} \frac{J_{\mathrm{c}}^{2}}{T^{2}}+\ldots
$$

written here in units 'natural' such that $k_{\mathrm{B}}=1$. It also controls the deviation of the high temperature magnetic susceptibility from a simple Curie law

$$
\chi^{-1}(T \rightarrow \infty)=\frac{T+\Theta_{\mathrm{CW}}+\frac{1}{2} \frac{J_{\mathrm{c}}^{2}}{T}+\ldots}{C}
$$

where we again work in 'natural' units such that $\left(g \mu_{\mathrm{B}}\right)^{2}=1$,

$$
C=\frac{S(S+1)}{3}=\frac{1}{4}
$$

and

$$
\Theta_{\mathrm{CW}}=4\left(J_{1}+J_{2}\right) C=J_{1}+J_{2} .
$$

However, while knowledge of both $J_{\mathrm{c}}$ and $\Theta_{\mathrm{CW}}$ fixes $J_{1}+J_{2}$ and $\left|J_{1}-J_{2}\right|$, the sign of $J_{1}-J_{2}$ remains undetermined since there are two possible values $\phi_{ \pm}$of the angle $\phi$. And because $\phi$ determines the physics of the $J_{1}-J_{2}$ model, this uncertainty can lead to alternative parameterizations of the model which lie in completely different phases. 


\begin{tabular}{c|cc|ccccc|ccc} 
& \multicolumn{7}{|c|}{$\mathrm{Pb}_{2} \mathrm{VO}\left(\mathrm{PO}_{4}\right)_{2}$} & \multicolumn{5}{|c|}{$\mathrm{Li}_{2} \mathrm{VOSiO}_{4}$} & \multicolumn{4}{c}{$\mathrm{Li}_{2} \mathrm{VOGeO}_{4}$} \\
& {$[6]$} & & {$[4]$} & {$[5]$} & {$[18]$} & {$[19]$} & & {$[4]$} & {$[18]$} & \\
\hline$\Theta_{\mathrm{CW}}[\mathrm{K}]$ & 4 & & 7.4 & 8.2 & 9.65 & 7.2 & & 5.2 & 9.8 & \\
$\Theta_{\mathrm{CW}} / T_{\chi}$ & 0.49 & & 1.39 & 1.69 & & & & 1.49 & & \\
$\phi_{-} / \pi$ & 0.67 & 0.64 & 0.41 & 0.27 & 0.47 & 0.43 & 0.36 & 0.38 & 0.43 & 0.33 \\
$\phi_{+} / \pi$ & & -0.11 & 0.03 & 0.13 & & & 0.06 & 0.08 & & 0.07 \\
$\left(J_{2} / J_{1}\right)_{-}$ & -1.64 & -2.21 & 3.5 & 1.1 & 11.7 & 4.76 & 2.13 & 2.5 & 4.76 & 1.69 \\
$\left(J_{2} / J_{1}\right)_{+}$ & & -0.37 & 0.1 & 0.44 & & & 0.18 & 0.25 & & 0.24
\end{tabular}

Table 3. Compilation of the experimental results and theoretical estimates on the Curie-Weiss temperature $\Theta_{\mathrm{CW}}=\left(J_{1}+J_{2}\right) / k_{\mathrm{B}}$, the ratio $\Theta_{\mathrm{CW}} / T_{\chi}$ of it to the maximum position of the uniform magnetic susceptibility $\chi(T)$ and the corresponding frustration parameters. The data are taken from [4, 5, 6]. The displayed theoretical values for the frustration parameters obtained by fits to high-temperature series expansions are taken from [18, 19]. The reference numbers are used to label the corresponding columns. The unlabelled columns contain our own estimates derived from the dependence of $\Theta_{\mathrm{CW}} / T_{\chi}$ on $\phi$, see Figure 15 The \pm subscripts of $\phi$ and $J_{2} / J_{1}$ distinguish the two different possible points in the $\left(J_{1}, J_{2}\right)$ phase diagram.

The coefficients of the high temperature series expansions of the heat capacity and susceptibility $J_{1}-J_{2}$ model are known to high order [18, 26], and their reliability at low temperatures can be greatly improved by carefully constraining the analytic continuation of the high temperature series [19, 27]. None the less it proves very difficult to determine $J_{1}$ and $J_{2}$ unambiguously from experimental measurements of heat capacity and magnetic susceptibility. In the case of $\mathrm{Li}_{2} \mathrm{VOSiO}_{4}$, where both $J_{1}$ and $J_{2}$ are believed to be antiferromagnetic, estimates of the ratio of $J_{2} / J_{1}$ vary by more than a factor ten [4, 5, 18, 19, 26], see Table 3 Preliminary analysis of $\mathrm{Pb}_{2} \mathrm{VO}\left(\mathrm{PO}_{4}\right)_{2}$, where either $J_{1}$ or $J_{2}$ is believed to be ferromagnetic, does not unambiguously determine which is the ferro- and which the antiferromagnetic coupling [6].

In the light of this uncertainty and controversy, we have used our analytic solution of the 8-site cluster and an implementation of the finite temperature Lanczos method (FTLM) to determine the heat capacity, uniform magnetic susceptibility and static spin structure factor for the model on 16- and 20 -site clusters with periodic boundary conditions. In particular, below, we present predictions for diffuse neutron scattering cross sections which are difficult to access by series expansion, and which can in principle remove all ambiguity about the parameterization of the model.

Since these calculations are based on small clusters of spins, they are of limited use in addressing questions such as the finite temperature Ising transition observed in Monte Carlo simulations of the classical square-lattice $J_{1}-J_{2}$ model [28]. However they should provide a reliable guide to physics at finite temperatures and short length scales, particularly in the frustrated phases of the model, where long range order is greatly suppressed.

The FTLM is based on the Lanczos algorithm which is used to iteratively exactly diagonalize the Hamiltonian matrix for the cluster considered: The Lanczos algorithm starts with a randomly chosen vector in the Hilbert space. Successive applications of the Hamiltonian eventually "rotates" the starting vector to the ground state of the system (if the starting vector is not orthogonal to it), thereby generating a tridiagonal matrix having eigenvalues and eigenvectors corresponding to the lowest eigenvalues and eigenvectors of the full Hamiltonian (this always has to be checked). The FTLM utilizes the eigenvalues and eigenvectors of 1000 successive diagonalizations with dif-

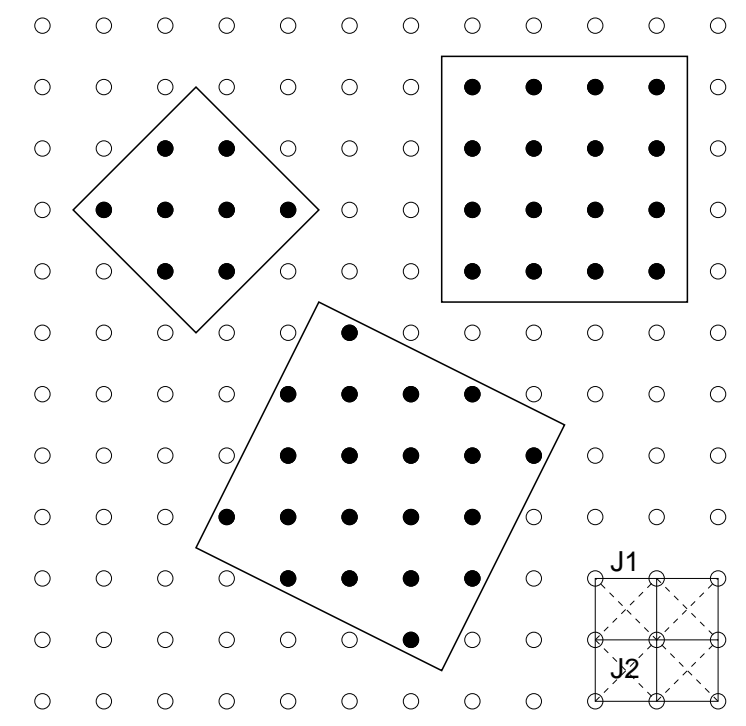

Fig. 14. Tiles of size eight, 16 , and 20 used in the finite-temperature calculations. In the lower right corner, the labelling of the two exchange constants is illustrated.

ferent random starting vectors to generate the partition function and the expectation values as defined below. For a detailed description of it, see [29].

The three clusters, together with the realisation of the spin exchange interactions, are shown in Figure 14 Due to the symmetry of the model, these are the only possible squares of size $N$ with $4<N<32$ which are compatible with both collinear and Néel order. With three cluster sizes at hand, knowing that the eight-site cluster is almost a complete graph and the 16site cluster a four-dimensional hypercube, we did not attempt to perform a finite-size scaling analysis. For this to be meaningful, results for a system size at least $N=32$, and as well as for different (i. e. open) boundary conditions would be needed. 


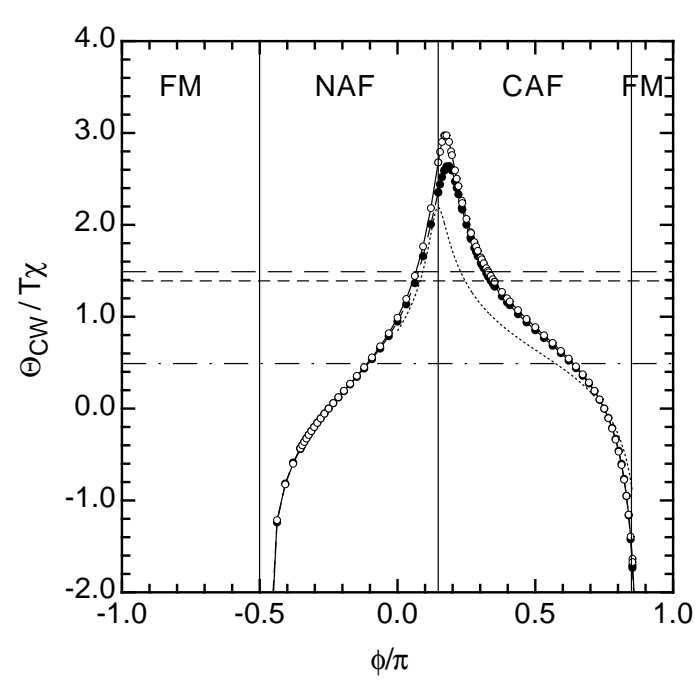

Fig. 15. Ratio of the Curie-Weiss temperature $\Theta_{\mathrm{CW}}$ to the maximum position $T_{\chi}$ of the uniform magnetic susceptibility $\chi(T)$ as a function of the frustration angle $\phi$. The solid (dashed) line denote the results for the 20-site (16-site) cluster, the dotted line comprises the result for the eight-site cluster. The straight horizontal lines correspond to the experimental values $\Theta_{\mathrm{CW}} / T_{\chi}=0.49,1.39,1.49$ for $\mathrm{Pb}_{2} \mathrm{VO}\left(\mathrm{PO}_{4}\right)_{2}$ (dash-dotted) [6], $\mathrm{Li}_{2} \mathrm{VOSiO}_{4}$ (dashed) [4], and $\mathrm{Li}_{2} \mathrm{VOGeO}_{4}$ (longdashed) [4], respectively.

\subsection{Heat capacity and magnetic susceptibility}

In units with dimensions restored, the heat capacity and the magnetic susceptibility are defined by

$$
\begin{aligned}
\chi(T) & =\frac{N_{\mathrm{A}} \mu_{0} g^{2} \mu_{\mathrm{B}}^{2}}{N k_{\mathrm{B}}} \frac{1}{T}\left(\left\langle\left(S_{z}^{\mathrm{tot}}\right)^{2}\right\rangle-\left\langle S_{z}^{\mathrm{tot}}\right\rangle^{2}\right), \\
C_{V}(T) & =\frac{N_{\mathrm{A}}}{N k_{\mathrm{B}}} \frac{1}{T^{2}}\left(\left\langle H^{2}\right\rangle-\langle H\rangle^{2}\right),
\end{aligned}
$$

where $\langle\ldots\rangle$ denotes the thermal average, $S_{z}^{\mathrm{tot}=\sum_{\mathrm{i}} \mathrm{S}_{\mathrm{i}}^{\mathrm{z}}}$ the $z$ component of the total momentum of the system, and $N$ the number of sites of the system considered. $N_{\mathrm{A}}$ is the Avogadro constant, $\mu_{0}$ the magnetic permeability, $g$ the gyromagnetic ratio, $\mu_{\mathrm{B}}$ the Bohr magneton, and $k_{\mathrm{B}}$ the Boltzmann constant. For the present nonmagnetic (zero field) case we have $\left\langle S_{z}^{\text {tot }}\right\rangle=0$.

A commonly used experimentally accessible parameter to determine the frustration angle $\phi$ (and hence the value $J_{2} / J_{1}$ ) is the ratio of the position $T_{\chi}$ of the maximum of the magnetic susceptibility $\chi(T)$ to the Curie-Weiss temperature $\Theta_{\mathrm{CW}}$. To avoid singularities, we plot the inverse of this quantity, which is shown in Figure 15

Apart from the strongly frustrated spin-liquid region around $\phi \approx \pi / 6$, the differences in $\Theta_{\mathrm{CW}} / T_{\chi}$ for the 16 - and 20 -site cluster are small. This suggests that the behaviour of $\chi(T)$, at least for $k_{\mathrm{B}} T \geq J_{\mathrm{c}}$, is dominated by correlations which are fully taken into account already by the small clusters, and therefore finite-size effects do not play an important role.

A common feature of $\Theta_{\mathrm{CW}} / T_{\chi}$ for all cluster sizes is the existence of two possible angles $\phi_{-}$and $\phi_{+}$for a given value for $\Theta_{\mathrm{CW}} / T_{\chi}$, reflecting the fact that from the knowledge of $\Theta_{\mathrm{CW}}$ and $J_{\mathrm{c}}$ alone, $\phi$ cannot be determined unambiguously. We have indicated the experimental values taken from [4] and [6] for $\Theta_{\mathrm{CW}} / T_{\chi}$ for $\mathrm{Pb}_{2} \mathrm{VO}\left(\mathrm{PO}_{4}\right)_{2}, \mathrm{Li}_{2} \mathrm{VOSiO}_{4}$, and $\mathrm{Li}_{2} \mathrm{VOGeO}_{4}$ by the thin horizontal lines in Figure 15 For all three compounds, $\phi$ - corresponds to a realization of the phase with strong collinear antiferromagnetic correlations, and $\phi_{+}$to the phase where Néeltype correlations dominate. Interestingly,

some values of $\phi_{+}$lie very close to the spin-liquid regime $0.115<\phi / \pi<0.183$.

Table 3 holds a summary of the values for $\Theta_{\mathrm{CW}} / T_{\chi}$ and $\phi_{ \pm}=\tan ^{-1}\left(\left(J_{2} / J_{1}\right)_{ \pm}\right)$found in the literature [4, 5, 6, 18, 19], together with our findings. $\Theta_{\mathrm{CW}}=\left(J_{1}+J_{2}\right) / k_{\mathrm{B}}$ can be determined by a fit of a Curie-Weiss law to the high-temperature tail of the susceptibility $\chi(T)$, as was done for the two Li compounds [4, 5]. This procedure can be improved by including higher-order terms in an expansion of $\chi(T)$, see equation (32). For the $\mathrm{Pb}$ compound [6], the high-temperature series expansion for $\chi(T)$ found in [18] was applied. For the compound $\mathrm{Li}_{2} \mathrm{VOSiO}_{4}$ [4, 5], this leads to errors of the order of $10 \%$.

In contrast to $\Theta_{\mathrm{CW}}$, the determination of the frustration angle $\phi$ is much more involved. Melzi et al. [4, 5] use exact diagonalization data for the heat capacity of the eight- and 16-site clusters [30, 31], while again fits to high-temperature series expansions are used in [6, 18, 19]. In particular for $\mathrm{Li}_{2} \mathrm{VOSiO}_{4}$, the results for $J_{2} / J_{1}$ differ by more than an order of magnitude. Still they indicate qualitatively the same ordered phase in the ground state of the compound.

However, $J_{1}$ and $J_{2}$ are not uniquely determined by this analysis of the temperature dependence of the magnetic susceptibility or the comparison with the behaviour of the specific heat alone. We will return to this issue in the next section discussing the static spin structure factor.

We have computed the heat capacity $C_{V}(T)$ in the full range of the frustration angle $\phi$ for different cluster sizes. Figure 16 shows the maximum of the heat capacity as a function of $\phi$. The bottom part of the figure shows the frustration dependence of the temperature $T_{C_{V}}$ at which the maximum is reached.

Two overall effects are clearly visible: (1) Apart from the regime with strong frustration, the maximum increases with increasing cluster size. (2) The maximum temperature decreases with increasing cluster size. Taken together, this indicates that entropy is shifted to lower temperatures, a sign of the missing long-range correlations not included in the partition function for the small clusters.

Our results are in qualitative agreement with those in 5 , 19]. They represent a quantitative improvement over the estimates of [5]. Direct comparison with [19] is made difficult by the ambiguities associated with analytic continuation of a series using Padé approximants, and by the fact that the limited number of cluster sizes we can use at present do not permit a finite size scaling analysis. In agreement with [5], $C_{V}^{\max }$ drops sharply near the crossover between the spin liquid regime and the collinear phase around $J_{2} / J_{1} \approx 0.6$, corresponding to $\phi / \pi \approx 0.17$. Similar drops occur at the borders of the FM regime with the NAF and CAF phases, respectively. These drops are accompanied with a smaller $T_{C_{V}}$ in order to conserve the entropy of the system.

In Figure 17 the behaviour of the maximum of the magnetic susceptibility $\chi^{\max }$ together with the temperature at which the maximum is reached is displayed. In contrast to the heat 

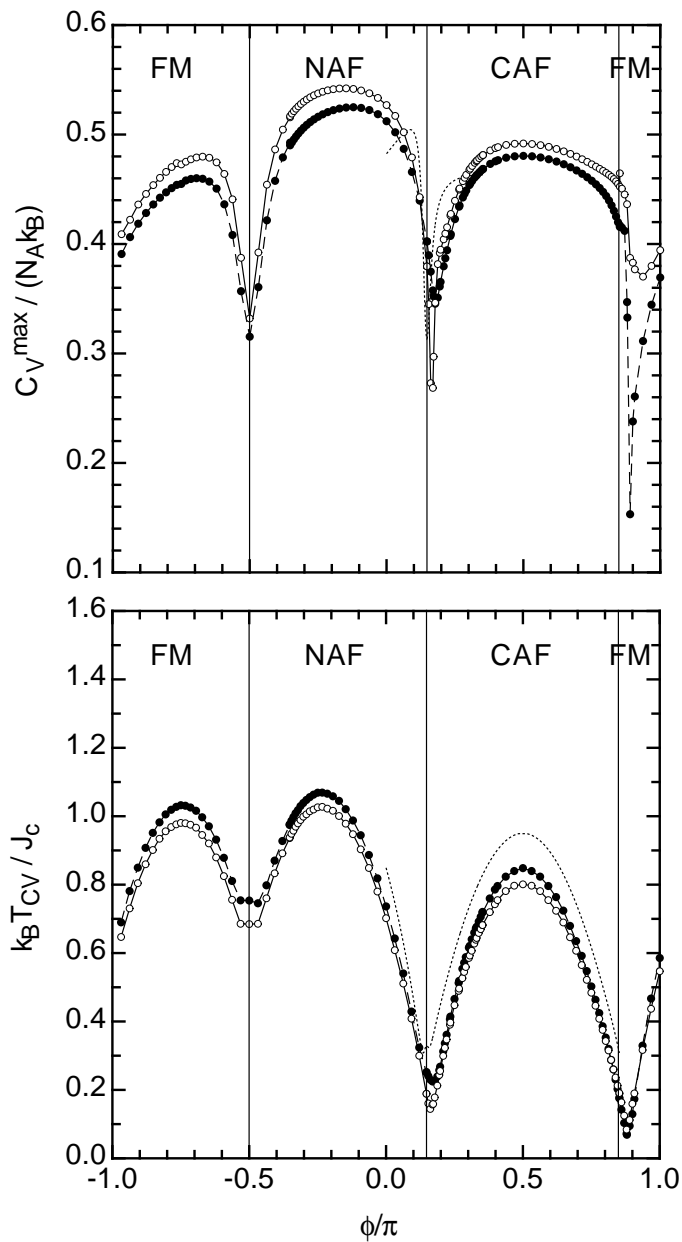

Fig. 16. Maximum of the heat capacity $C_{V}(T)$ and its position $T_{C_{V}}$ as functions of the frustration angle $\phi$. The open (solid) circles denote the results for the 20-site (16-site) cluster, the dotted line denotes the eight-site cluster.

capacity, $\chi(T)$ does not display an anomaly upon crossing the spin-liquid regime. The maximum value diverges near the crossove to the FM regime, while its position approaches $T=0$, which is the expected behaviour. Apart from that, the parameter dependence of the maximum position $T_{\chi}$ is qualitatively the same as for $T_{C_{V}}$.

Due to the sharp drop of the maximum of the heat capacity, the ratio of the two temperatures $T_{\chi}$ and $T_{C_{V}}$ shows a pronounced anomaly which has a strong dependence on cluster size in the spin liquid regime at $\phi / \pi \approx 0.17\left(J_{2} / J_{1} \approx 0.6\right)$. In Figure 18, we have plotted $T_{\chi} / T_{C_{V}}$ as a function of $\phi$. For $0.3 \leq \phi / \pi \leq 0.85$, i. e., in the collinear phase, $T_{\chi} / T_{C_{V}}$ does not depend on $\phi$. For negative values $-1 / 2<\phi / \pi \leq-0.15$ (in the Néel phase), $T_{\chi} / T_{C_{V}}$ depends roughly linearly on the frustration angle, providing an additional means to determine this angle uniquely from thermodynamic measurements alone. (Unfortunately, none of the three compounds discussed in this paper fall into this category.)
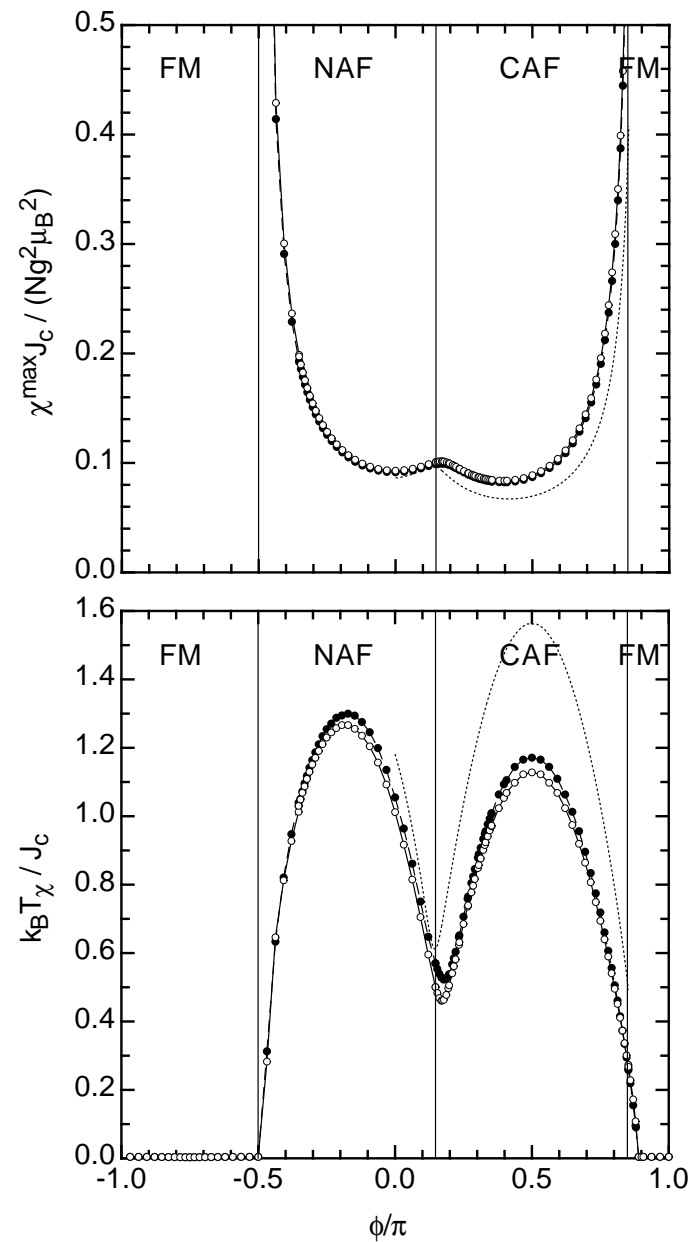

Fig. 17. Maximum of the uniform magnetic susceptibility $\chi(T)$ and its position $T_{\chi}$ as functions of the frustration angle $\phi$. The open (solid) circles denote the results for the 20 -site (16-site) cluster, the dotted line denotes the eight-site cluster.

\begin{tabular}{c|cccccc}
$\mathbf{q}$ & $(0,0)$ & $\left(\frac{\pi}{2}, 0\right)$ & $(\pi, 0)$ & $\left(\pi, \frac{\pi}{2}\right)$ & $(\pi, \pi)$ & $\left(\frac{\pi}{2}, \frac{\pi}{2}\right)$ \\
\hline $\mathrm{m}$ & 1 & 4 & 2 & 4 & 1 & 4
\end{tabular}

Table 4. List of momentum vectors $\mathbf{q}$ for the 16-site square together venith their multiplicity $m$.

\subsection{Spin structure factor}

The finite temperature Lanczos approach also permits the direct evaluation of correlation functions. We consider here the static spin structure factor given by

$$
S(\mathbf{q}, T)=\frac{1}{N} \sum_{i, j=1}^{N} e^{\mathrm{iq}\left(\mathbf{R}_{i}-\mathbf{R}_{j}\right)}\left\langle\mathbf{S}_{i} \mathbf{S}_{j}\right\rangle
$$

We have calculated the temperature dependence of the spinspin correlation functions $\left\langle\mathbf{S}_{i} \mathbf{S}_{j}\right\rangle$ for the 16-site cluster and performed the necessary summations to determine $S(\mathbf{q}, T)$. The irreducible triangle of the Brillouin zone of the 16-site cluster contains six points which are listed together with their multi- 


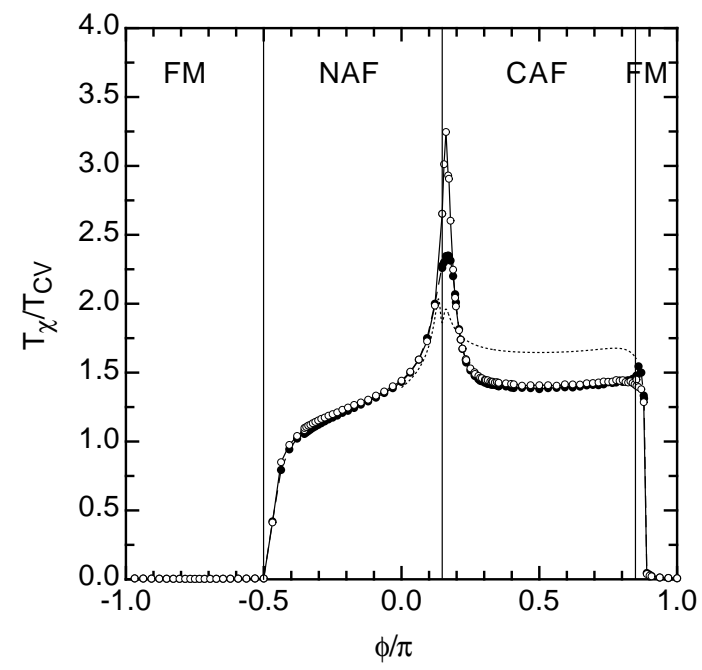

Fig. 18. Ratio $T_{\chi} / T_{C_{V}}$ of the maximum positions of the uniform magnetic susceptibility $\chi(T)$ and the heat capacity $C_{V}(T)$ as a function of the frustration angle $\phi$. The solid (dashed) lines denote the results for the 20-site (16-site) cluster, the dotted line denotes the eight-site cluster.

plicity in table 4 All points lie on the edges and corners of the triangle.

In Figure 19 $S(\mathbf{q})$ is displayed as a function of $\mathbf{q}$ lying on the edge of the irreducible Brillouin zone triangle. We have chosen $J_{1} / k_{\mathrm{B}}=1.25 \mathrm{~K}, J_{2} / k_{\mathrm{B}}=5.95 \mathrm{~K}$ (collinear phase) and $J_{1} / k_{\mathrm{B}}=5.95 \mathrm{~K}, J_{2} / k_{\mathrm{B}}=1.25 \mathrm{~K}$ (Néel phase). These values for $J_{1}$ and $J_{2}$ correspond to those found for $\mathrm{Li}_{2} \mathrm{VOSiO}_{4}$ in [19]; the former two correspond to $\phi_{-} / \pi=0.43$, the latter have a frustration angle of $\phi_{+} / \pi=0.07$. The dots represent the numerical results; the lines connecting them are just guides to the eye. We plot $S(\mathbf{q}, T)$ for ten different temperatures $k_{\mathrm{B}} T / J_{\mathrm{c}}=$ $1,2,3 \ldots 10$ with an offset of one half between each two curves.

For $\mathrm{Pb}_{2} \mathrm{VO}\left(\mathrm{PO}_{4}\right)_{2}$, currently no suitable single crystals are available to be able to measure $S(\mathbf{q}, T)$ by diffuse neutron scattering. Therefore, we have calculated the angular average over the momentum transfer of $S(\mathbf{q}, T)$, which can be experimentally determined using powder or polycrystalline material. The results are displayed in Figure 20 as $S(|\mathbf{q}|, T)$ versus the modulus $q=|\mathbf{q}|$ of the momentum transfer and the temperature $T$. We have chosen the two frustration angles $\phi_{-}=0.64$ where the system is in the collinear phase and $\phi_{+}=-0.11$ corresponding to the Néel phase. For the former, the maximum of $S(|\mathbf{q}|, T)$ is located at $|\mathbf{q}|=\pi$, the latter reaches its maximum near the zone boundary where $|\mathbf{q}|=\sqrt{2} \pi$.

To summarize, from Figures 19 and 20 we can conclude that the structure factor provides a means to determine the frustration ratio $\phi$ and therefore the ordering wave vector unambiguously: For $\phi_{-}, S(\mathbf{q})$ is strongly peaked at $\mathbf{q}=(\pi, 0)$ or $(0, \pi)$, while for $\phi_{+}$it is peaked at $\mathbf{q}=(\pi, \pi)$ as one would expect from the associated broken symmetries for $\phi_{ \pm}$in the thermodynamic limit.

Figure 21 displays the temperature dependence of $S(\mathbf{q}, T)$ for different values of $\mathbf{q}$. In the collinear and the Néel phase, at low temperatures, $S(\mathbf{q}, T)$ develops a pronounced anomaly at the ordering vector $\mathbf{Q}$ characterizing the phase. This anomaly
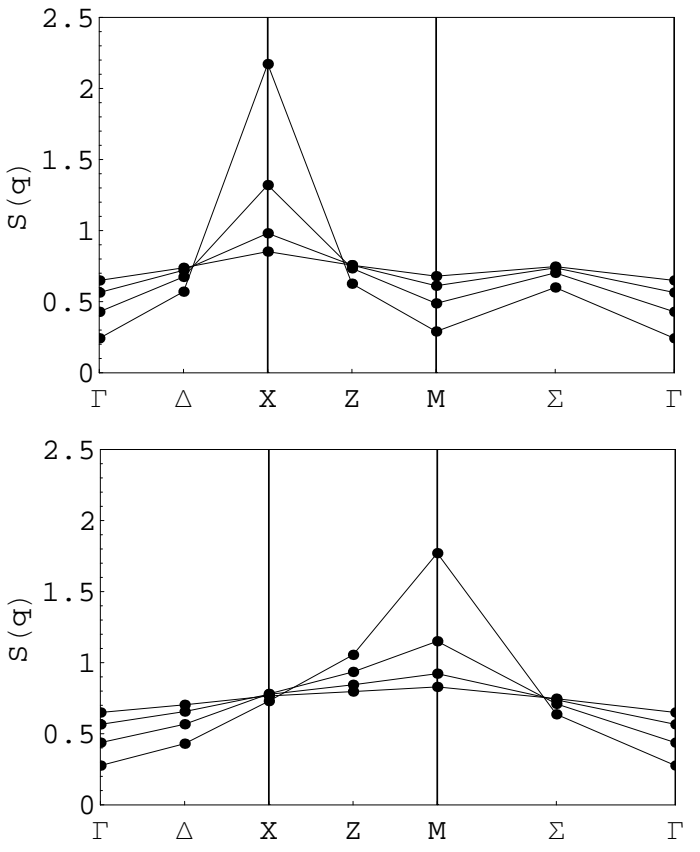

Fig. 19. Static spin structure factor $S(\mathbf{q}, T)$ of the 16-site cluster for $J_{1} / k_{\mathrm{B}}=1.25 \mathrm{~K}, J_{2} / k_{\mathrm{B}}=5.95 \mathrm{~K}$ (collinear phase, top) and $J_{1} / k_{\mathrm{B}}=$ $5.95 \mathrm{~K}, J_{2} / k_{\mathrm{B}}=1.25 \mathrm{~K}$ (Néel phase, bottom figure). The values chosen for $J_{1}$ and $J_{2}$ correspond to those determined for $\mathrm{Li}_{2} \mathrm{VOSiO}_{4}$ in [19]. The lines are guides to the eye, the dots denote the numerical results. The individual curves in each figure, from bottom to top at $\mathbf{q}=\Gamma$, correspond to fixed temperatures $k_{\mathrm{B}} T / J_{\mathrm{c}}=1,2,4$, and 8 .

is the precursor of a divergence of $S(\mathbf{q}, T \rightarrow 0)$ for the infinite system. The asymptotic value for $k_{\mathrm{B}} T / J_{\mathrm{c}} \rightarrow \infty$ is $S(\mathbf{q})=S(S+$ $1)=3 / 4$ in each case, as it should be.

In the collinear as well as in the Néel phase, the relation $S(\mathbf{Q})>S(\mathbf{q}), \mathbf{q} \neq \mathbf{Q}$ holds for all temperatures, while for the spin liquid regime, this is qualitatively different: Here, the value of $S(\mathbf{q}, T)$ is approximately the same for $\mathbf{q}=(\pi, \pi)$ and $\mathbf{q}=$ $(\pi, 0)$ or $(0, \pi)$ at temperatures $k_{\mathrm{B}} T>J_{\mathrm{c}}$ and always larger than the value for $\mathbf{q}=0$, which is an additional indicator for the strong frustration in that phase. The approximate equality of $S(\mathbf{q}, T)$ values for different wave vectors also supports the picture that close to the SL phase domains of the CAF phase may easily form inside the NAF and vice versa as illustrated in Figure 3

To illustrate our numerical findings further, we have taken the Fourier transform of the high-temperature series expansion for the structure factor up to first order using the result in 32 , page 709ff] 

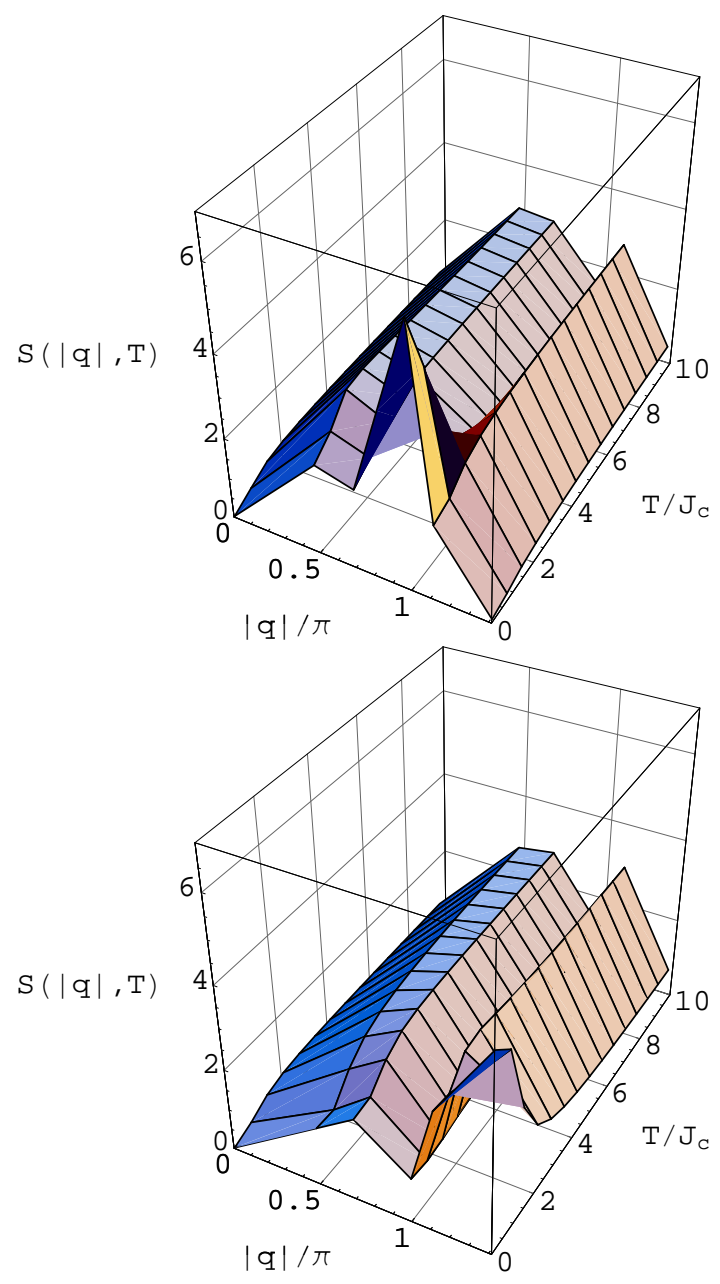

Fig. 20. Static spin structure factor $S(|\mathbf{q}|, T)$ of the 16-site cluster for $J_{1} / k_{\mathrm{B}}=-6 \mathrm{~K}, J_{2} / k_{\mathrm{B}}=10 \mathrm{~K}$ (collinear phase, top) and $J_{1} / k_{\mathrm{B}}=10 \mathrm{~K}$, $J_{2} / k_{\mathrm{B}}=-6 \mathrm{~K}$ (Néel phase, bottom figure). The values chosen for $J_{1}$ and $J_{2}$ correspond to those given for $\mathrm{Pb}_{2} \mathrm{VO}\left(\mathrm{PO}_{4}\right)_{2}$ in [6].

$$
\begin{array}{r}
S(\mathbf{q}, T)=S(S+1)+\frac{S_{1}(\mathbf{q}, \phi)}{k_{\mathrm{B}} T / J_{\mathrm{c}}}+\mathscr{O}\left(\frac{1}{k_{\mathrm{B}} T / J_{\mathrm{c}}}\right)^{2}, \\
S_{1}(\mathbf{q}, \phi)=-\frac{z S^{2}(S+1)^{2}}{3}\left(\cos \phi \frac{1}{2}\left(\cos q_{x}+\cos q_{y}\right)\right. \\
\left.+\sin \phi \cos q_{x} \cos q_{y}\right),
\end{array}
$$

where $z=4$ is the coordination number of the lattice. In Figure 21 the dash-dotted lines in the three panels for the different regimes in the $\left(J_{1}, J_{2}\right)$ phase diagram represent the first two terms of equation (38) for the corresponding characteristic wave vectors $\mathbf{q}=(\pi, 0)$ or $(0, \pi)$ in the collinear phase, $\mathbf{q}=(\pi, \pi)$ in the Néel phase, and both of these values in the spin liquid regime.

In the two phases where the system has a magnetically ordered ground state, the high-temperature approximation for $S(\mathbf{q})$ at the respective ordering vector already underestimates the exact-diagonalization results for temperatures $k_{\mathrm{B}} T / J_{\mathrm{c}} \approx 5$
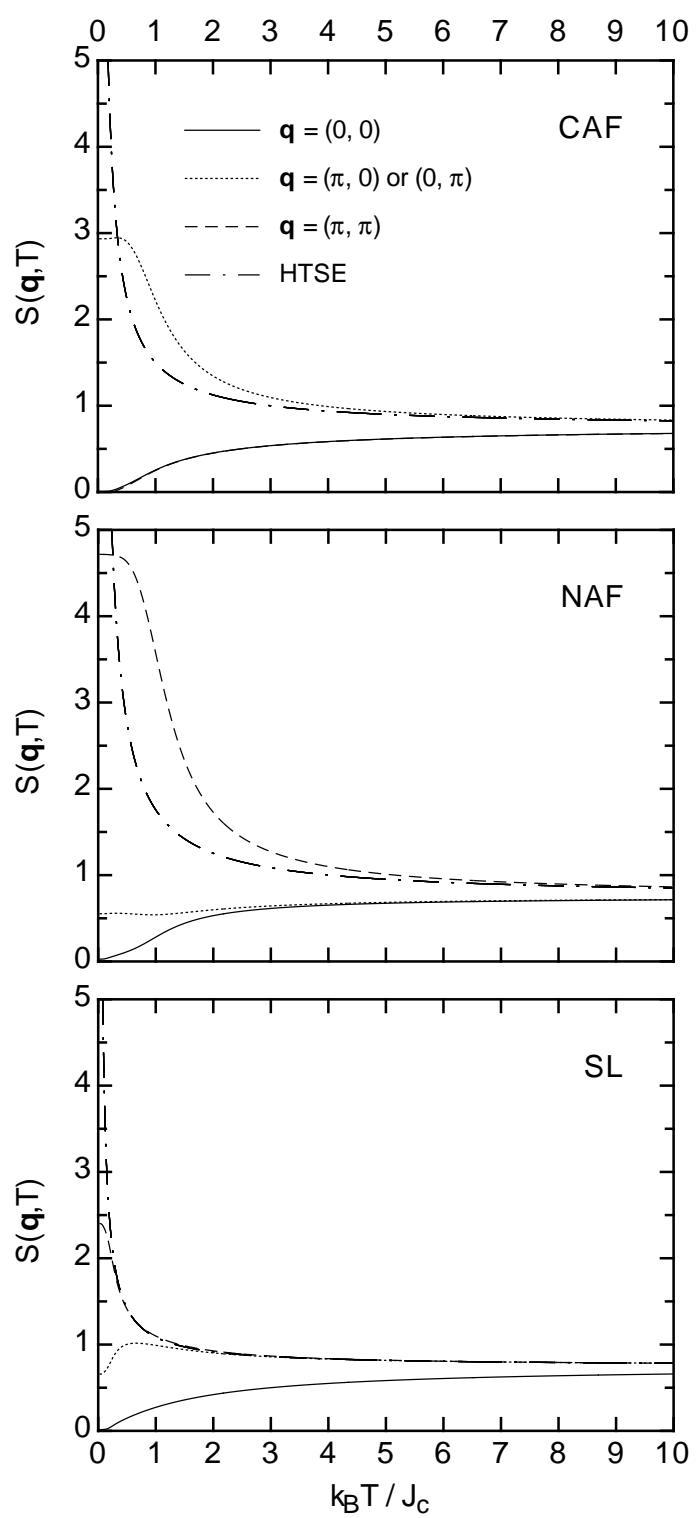

Fig. 21. Static spin structure factor $S(\mathbf{q}, T)$ as a function of temperature for $\mathbf{q}=(0,0)$ (solid line), $\mathbf{q}=(\pi, 0)$ or $(0, \pi)$ (dotted line), and $\mathbf{q}=(\pi, \pi)$ (dashed line) for three different frustration angles $\phi=\pi / 2$ (collinear phase, top), $\phi=\tan ^{-1}(-1 / 2)$ (Néel phase, middle), and $\phi=\tan ^{-1}(1 / 2)$ (spin liquid, SL, phase, bottom figure). The dashdotted lines in each of the three panels denote the high-temperature expansions (HTSE) of the structure factor at the respective value for $\mathbf{q}$ where $S(\mathbf{q}, T \rightarrow 0)$ diverges, i.e., from top to bottom $\mathbf{q}=(\pi, 0)$ for the collinear antiferromagnet, $\mathbf{q}=(\pi, \pi)$ for the Néel phase, and $\mathbf{q}=(\pi, 0)$ or $(\pi, \pi)$ in the spin liquid regime.

and below. In contrast, for the spin liquid regime, $S(\mathbf{q})$ is well reproduced for temperatures $k_{\mathrm{B}} T$ well below $J_{\mathrm{c}}$, demonstrating that long-range correlation effects are suppressed due to the presence of the strong frustration. From equation 38 we can also conclude that at high temperatures $k_{\mathrm{B}} T \gg J_{\mathrm{c}}$ the relation $S(\mathbf{q}=(\pi, 0))=S(\mathbf{q}=(\pi, \pi))$ holds exactly for $J_{2} / J_{1}=1 / 2$. We have chosen this particular frustration ratio for the lower panel in Figure 21 


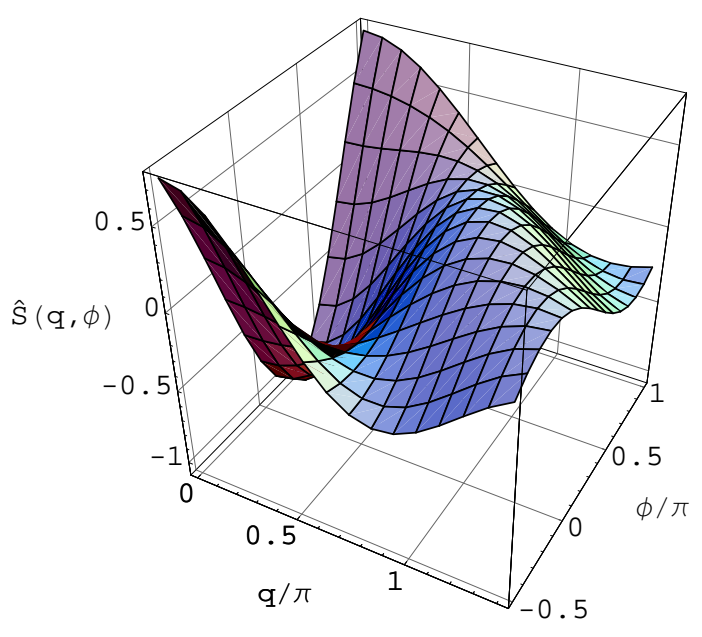

Fig. 22. Angular average $\hat{S}(q, \phi)$ of the first-order term of the hightemperature series expansion of the static spin structure factor $S(\mathbf{q}, T)$ as a function of the modulus $q$ of the momentum transfer and the frustration angle $\phi=-\pi / 2 \ldots \pi . \phi=-\pi / 2$ corresponds to $J_{1}=0$, $J_{2} / J_{\mathrm{c}}=-1$, which is the border between the ferromagnetic and the Néel phase in Figure $1 \phi=\pi$ corresponds to $J_{1} / J_{\mathrm{c}}=-1$ and $J_{2}=0$. Going from $\phi=-\pi / 2$ to $\phi=\pi$, we successively scan the Néel phase, the spin liquid regime, the collinear and finally the ferromagnetic phase (see text).

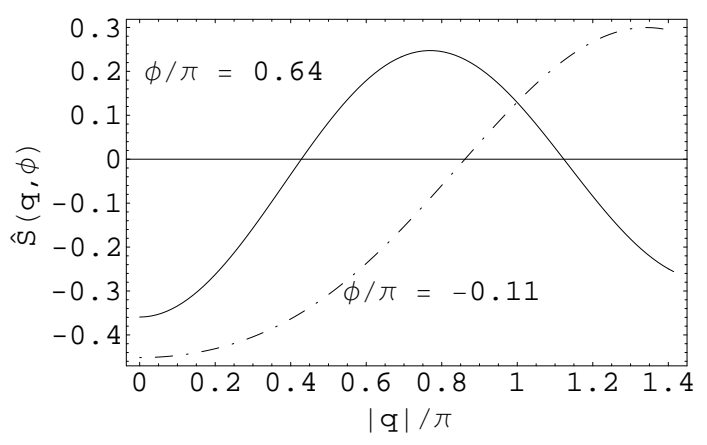

Fig. 23. Same as Figure 22 here for the two fixed values $\phi=\phi_{ \pm}$for $\mathrm{Pb}_{2} \mathrm{VO}\left(\mathrm{PO}_{4}\right)_{2}$ (Table 3). Solid line: $\phi / \pi=\phi_{-} / \pi=0.64$ (collinear phase), dash-dotted line: $\phi / \pi=\phi_{+} / \pi=-0.11$ (Néel phase).

In Figures 22 and 23, we have plotted the angular average $\hat{S}(q, \phi)$ of the first-order term of the high-temperature series expansion, equation (39), given by

$$
\hat{S}(q, \phi)=\frac{1}{2 \pi} \int_{0}^{2 \pi} \mathrm{d} \alpha S_{1}(\mathbf{q}, \phi), \quad \mathbf{q}=q(\cos \alpha, \sin \alpha) .
$$

Figure 22 demonstrates how the maximum of this coefficient of the structure factor evolves as a function of $\phi$.

Figure 23 shows $\hat{S}(q, \phi)$ as a function of the modulus of the momentum transfer $q=|\mathbf{q}|$ for the two possible values $\phi_{ \pm}$of the frustration angle for $\mathrm{Pb}_{2} \mathrm{VO}\left(\mathrm{PO}_{4}\right)_{2}$. The maximum for $\phi_{+}$ is near the zone boundary, whereas the maximum for $\phi_{-}$occurs at smaller values for $q$, confirming the conclusions of our exact-diagonalization results. Hence, for $k_{\mathrm{B}} T \gg J_{\mathrm{c}}$, it is possible to determine experimentally the correct value of $\phi$ from the quantity $\hat{S}(q, \phi) \approx k_{\mathrm{B}} T / J_{\mathrm{c}}(S(q, T)-S(S+1))$, and therefore together with the temperature dependences of $\chi(T)$ and $C_{V}(T)$ the exchange parameters $J_{1}$ and $J_{2}$.

\section{Summary and Conclusions}

Motivated by the discovery of $\mathrm{Pb}_{2} \mathrm{VO}\left(\mathrm{PO}_{4}\right)_{2}$, a " $J_{1}-J_{2}$ compound" with at least one FM $J$, we have extended the semiclassical description of the $J_{1}-J_{2}$ model to the case with FM couplings. We discussed the possible nature of the phase transitions between the three dominant phases, FM, NAF and CAF. On the basis of our results, the transition from CAF to FM seems to have much in common with the transition from CAF to NAF, where an intermediate spin liquid region is known to occur.

In addition to the phase diagram, the finite temperature properties of the model were discussed in the light of numerical results for 16 and 20 site clusters, using a newly implemented finite temperature Lanczos algorithm. In particular, we used this to calculate spin-spin correlation functions at high temperature, which can be compared with diffuse neutron scattering experiments. Our numerical results should be of use in resolving the controversy which surrounds parametrising $J_{1}-J_{2}$ compounds.

We also constructed a simple "tetragonal" mean field theory for the $J_{1}-J_{2}$ model, and performed an exact analytic diagonalization of an 8-site cluster. While these cannot be relied upon for quantitative comparison with experiment, they can be used to fit susceptibility data very easily, and seem to capture the essential physics of the model. Both are discussed in the Appendix.

Much of the most interesting magnetic physics occurs in frustrated FM's - He III on graphoil and the CMR manganites, to name but two - and it is our belief that the $J_{1}-J_{2}$ model deserves further study as such.

It is our pleasure to acknowledge helpful conversation with Noburo Fukushima, Christoph Geibel, George Jackeli, Enrique Kaul, R. Melzi, Gregoire Misguich, Philippe Sindzingre and Mike Zhitomirsky. We are particularly grateful to Michel Roger for extensive discussions and for providing us with the exact diagonalization code used to check the finite temperature Lanczos results.

This work was supported by the visitors programs of the Max Planck Institute for the Chemical Physics of Solids and the Max Planck Institute for the Physics of Complex Systems, and the following grants: Hungarian OTKA D32689, T037451 and the EU Center of Excellence ICA1-CT-2000-70029.

\section{References}

1. G. Misguich and C. Lhuillier (2003), cond-mat/0310405.

2. S. Sorella, Physical Review Letters 80, 4558 (1998).

3. P. Millet and C. Satto, Materials Research Bulletin 33, 1339 (1998).

4. R. Melzi, P. Carretta, A. Lascialfari, M. Mambrini, M. Troyer, P. Millet, and F. Mila, Physical Review Letters 85, 1318 (2000). 
5. R. Melzi, S. Aldrovandi, F. Tedoldi, P. Carretta, P. Millet, and F. Mila, Physical Review B 64, 024409 (2001).

6. E. E. Kaul, H. Rosner, N. Shannon, R. V. Shpanchenko, and C. Geibel, international Conference on Magnetism, Rome 2003; to be published.

7. J. Villain, Journal of Physics and Chemistry of Solids 11, 303 (1959).

8. R. R. P. Singh, Z. Weihong, C. J. Hamer, and J. Oitmaa, Physical Review B 60, 7278 (1999).

9. P. Chandra and B. Doucot, Physical Review B 38, 9335 (1988).

10. E. F. Shender, Soviet Physics - JETP 56, 178 (1982).

11. C. L. Henley, Physical Review Letters 62, 2056 (1989).

12. P. Chandra, P. Coleman, and A. I. Larkin, Physical Review Letters 64, 88 (1990).

13. J. Igarashi, Physical Review B 46, 10763 (1992).

14. G. Misguich, B. Bernu, C. Lhuillier, and C. Waldtmann, Physical Review Letters 81, 1098 (1998).

15. J. Fouet, P. Sindzingre, and C. Lhuillier, The European Physical Journal B 20, 241 (2001).

16. E. Dagotto and A. Moreo, Physical Review B 39, 4744 (1989)

17. O. P. Sushkov, J. Oitmaa, and Z. Weihong, Physical Review B 63, 104420 (2001).

18. H. Rosner, R. R. P. Singh, W. H. Zheng, J. Oitmaa, and W. E. Pickett, Physical Review B 67, 014416 (2003).

19. G. Misguich, B. Bernu, and L. Pierre, Physical Review B 68, 113409 (2003).

20. H. J. Schulz, T. A. L. Ziman, and D. Poilblanc, Journal De Physique I 6, 675 (1996).

21. P. W. Anderson, Physical Review 86, 694 (1952).

22. B. Bernu, C. Lhuillier, and L. Pierre, Physical Review Letters 69, 2590 (1992).

23. P. Azaria, B. Delamotte, and D. Mouhanna, Physical Review Letters 70, 2483 (1993).

24. E. Rastelli, L. Reatto, and A. Tassi, Journal of Physics C: Solid State Physics 19, 6623 (1986).

25. D. V. Dmitriev, V. Y. Krivnov, and A. A. Ovchinnikov, Physical Review B 55, 3620 (1997).

26. H. Rosner, R. P. Singh, W. H. Zheng, J. Oitmaa, S.-L. Drechsler, and W. E. Pickett, Physical Review Letters 88, 186405 (2002).

27. M. Roger, Physical Review B 58, 11115 (1998).

28. C. Weber, L. Capriotti, G. Misguich, F. Becca, M. Elhajal, and F. Mila, Physical Review Letters 91, 177202 (2003).

29. J. Jaklic and P. Prelovsek, Advances in Physics 49, 1 (2000).

30. R. R. P. Singh and R. Narayanan, Physical Review Letters 65, 1072 (1990).

31. S. Bacci, E. Gagliano, and E. Dagotto, Physical Review B 44, 285 (1991).

32. N. W. Ashcroft and N. D. Mermin, Solid State Physics (Saunders College Publishing, Philadelphia, 1988).

33. N. Shannon, The European Physical Journal B 27, 527 (2002).

34. M. V. D. Bossche, F.-C. Zhang, and F. Mila, European Physical Journal B 17, 367 (2000).

35. P. W. Anderson, Physical Review 83, 1260 (1951).
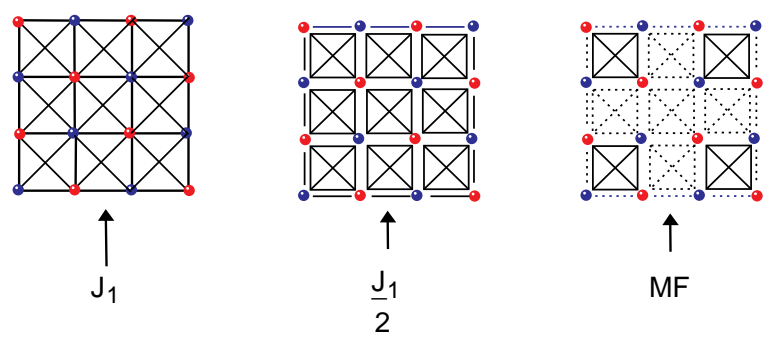

Fig. 24. The geometric content of the tetragonal mean-field theory for the $J_{1}-J_{2}$ model.

\begin{tabular}{ccccc}
\hline degeneracy & $\Omega_{+}$ & $\omega_{A}$ & $\omega_{B}$ & $E$ \\
\hline 1 & 2 & 1 & 1 & $J_{1} / 2+J_{2} / 2$ \\
1 & 1 & 1 & 0 & $-J_{2} / 2$ \\
1 & 1 & 0 & 1 & $-J_{2} / 2$ \\
1 & 1 & 1 & 1 & $-J_{1} / 2+J_{2} / 2$ \\
1 & 0 & 1 & 1 & $-J_{1}+J_{2} / 2$ \\
1 & 0 & 0 & 0 & $-3 J_{2} / 2$ \\
\hline
\end{tabular}

Table 5. The energy spectrum of a single tetrahedron.

36. H. Nishimori and Y. Ozeki, Journal of the Physical Society of Japan 58, 1027 (1989).

\section{A Tetragonal Mean field theory}

We take as a starting point the Hamiltonian in equation $(8)$ for a single tetrahedron (cross-linked square), and treat this fully connected 4-site cluster as a building block for the lattice. From knowledge of the spectrum and degeneracies given in Table 5 we can can calculate the partition function of a single tetrahedron exactly, and from that, its magnetic susceptibility and heat capacity [33].

Starting from knowledge of the exact susceptibility of a tetrahedron $\chi^{\mathrm{TET}}(T)$, we can construct a mean field theory for the lattice

$$
\chi_{\mathrm{MF}}(T)=\frac{\chi^{\mathrm{TET}}(T)}{1+3\left(J_{1}+J_{2}\right) \chi^{\mathrm{TET}}(T)}
$$

The geometric content of the mean-field approximation is explained in Figure 24 - one quarter of the tetrahedra making up lattice are treated exactly (double counting $J_{1}$ bonds), and the remaining bonds are treated on a mean-field level in such a way that each spin sees three exact and nine mean field bonds. Because of the very high degree of frustration involved, this approach should provide a reasonable account of the high temperature susceptibility when the ground state of the tetrahedron is a singlet, i. e. in the bulk NAF and CAF phases.

At high temperatures it is possible to expand the magnetic susceptibility found this way in $1 / T$, and to make a direct comparison with high temperature series expansions. Only the first two terms of equation (32) are reproduced exactly, but the functional form of $\chi(T)$ seems none the less to give a reasonably 


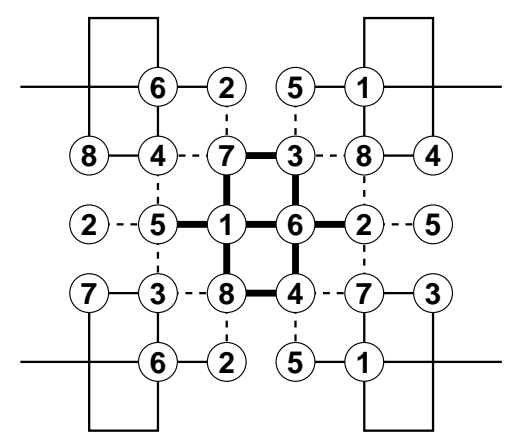

Fig. 25. The enumeration of sites used for the 8 -site cluster.

\begin{tabular}{|c|c|c|c|c|c|c|c|c|}
\hline deg. & $S_{1 \ldots 8}$ & $S_{1234}$ & $S_{12}$ & $S_{34}$ & $S_{5678}$ & $S_{56}$ & $S_{78}$ & $E$ \\
\hline 1 & 0 & 0 & 0 & 0 & 0 & 0 & 0 & 0 \\
\hline 2 & 0 & 0 & 0 & 0 & 0 & 1 & 1 & $-4 J_{2}$ \\
\hline 1 & 0 & 0 & 1 & 1 & 0 & 1 & 1 & $-8 J_{2}$ \\
\hline 4 & 0 & 1 & 0 & 1 & 1 & 0 & 1 & $-2 J_{1}$ \\
\hline 4 & 0 & 1 & 0 & 1 & 1 & 1 & 1 & $-2 J_{1}-2 J_{2}$ \\
\hline 1 & 0 & 1 & 1 & 1 & 1 & 1 & 1 & $-2 J_{1}-4 J_{2}$ \\
\hline 1 & 0 & 2 & 1 & 1 & 2 & 1 & 1 & $-6 J_{1}+4 J_{2}$ \\
\hline 4 & 1 & 0 & 0 & 0 & 1 & 0 & 1 & 0 \\
\hline 2 & 1 & 0 & 0 & 0 & 1 & 1 & 1 & $-2 J_{2}$ \\
\hline 4 & 1 & 0 & 1 & 1 & 1 & 0 & 1 & $-4 J_{2}$ \\
\hline 2 & 1 & 0 & 1 & 1 & 1 & 1 & 1 & $-6 J_{2}$ \\
\hline 4 & 1 & 1 & 0 & 1 & 1 & 0 & 1 & $-J_{1}$ \\
\hline 4 & 1 & 1 & 0 & 1 & 1 & 1 & 1 & $-J_{1}-2 J_{2}$ \\
\hline 4 & 1 & 1 & 0 & 1 & 2 & 1 & 1 & $-3 J_{1}+2 J_{2}$ \\
\hline 1 & 1 & 1 & 1 & 1 & 1 & 1 & 1 & $-J_{1}-4 J_{2}$ \\
\hline 2 & 1 & 1 & 1 & 1 & 2 & 1 & 1 & $-3 J_{1}$ \\
\hline 1 & 1 & 2 & 1 & 1 & 2 & 1 & 1 & $-5 J_{1}+4 J_{2}$ \\
\hline 2 & 2 & 0 & 0 & 0 & 2 & 1 & 1 & $2 J_{2}$ \\
\hline 2 & 2 & 0 & 1 & 1 & 2 & 1 & 1 & $-2 J_{2}$ \\
\hline 4 & 2 & 1 & 0 & 1 & 1 & 0 & 1 & $J_{1}$ \\
\hline 4 & 2 & 1 & 0 & 1 & 1 & 1 & 1 & $J_{1}-2 J_{2}$ \\
\hline 4 & 2 & 1 & 0 & 1 & 2 & 1 & 1 & $-J_{1}+2 J_{2}$ \\
\hline 1 & 2 & 1 & 1 & 1 & 1 & 1 & 1 & $J_{1}-4 J_{2}$ \\
\hline 2 & 2 & 1 & 1 & 1 & 2 & 1 & 1 & $-J_{1}$ \\
\hline 1 & 2 & 2 & 1 & 1 & 2 & 1 & 1 & $-3 J_{1}+4 J_{2}$ \\
\hline 4 & 3 & 1 & 0 & 1 & 2 & 1 & 1 & $2 J_{1}+2 J_{2}$ \\
\hline 2 & 3 & 1 & 1 & 1 & 2 & 1 & 1 & $2 J_{1}$ \\
\hline 1 & 3 & 2 & 1 & 1 & 2 & 1 & 1 & $4 J_{2}$ \\
\hline 1 & 4 & 2 & 1 & 1 & 2 & 1 & 1 & $4 J_{1}+4 J_{2}$ \\
\hline
\end{tabular}

Table 6. The energy spectrum of the 8 site cluster

good account of the experimental measured susceptibility. Since the partition function can be calculated for arbitrary magnetic field $h$, it is also easy to examine non-linear effects in $\chi(T, h)$. The 4-site cluster is however, too small to give a convincing description of $c_{V}(T)$.

\section{B Exact solution of the 8 site cluster}

In Section $\mathrm{A}$ we used the property that the energy spectrum of a complete graph depends only on the total spin to solve a fully connected 4-site cluster. We can use a generalization of the same trick [34] to solve the 8-site cluster with periodic boundary conditions's shown in Figure 25 Written in terms of complete graphs, the Hamiltonian of this cluster is:

$$
\begin{aligned}
\mathscr{H}_{8 \text { sites }}= & J_{1} \mathscr{H}_{12345678}^{\mathrm{CG}}+\left(2 J_{2}-J_{1}\right)\left(\mathscr{H}_{1234}^{\mathrm{CG}}+\mathscr{H}_{5678}^{\mathrm{CG}}\right) \\
& -2 J_{2}\left(\mathscr{H}_{12}^{\mathrm{CG}}+\mathscr{H}_{34}^{\mathrm{CG}}+\mathscr{H}_{56}^{\mathrm{CG}}+\mathscr{H}_{78}^{\mathrm{CG}}\right)
\end{aligned}
$$
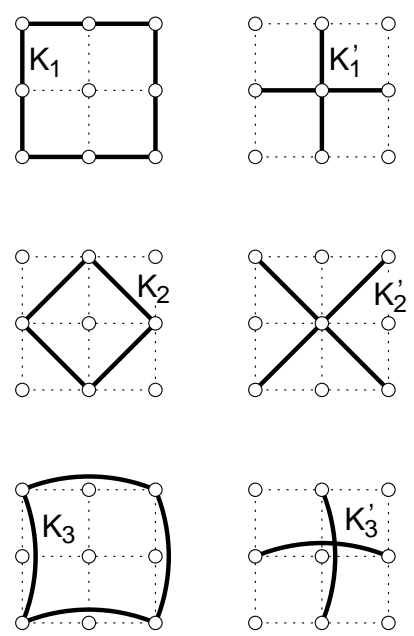

Fig. 26. The 9-site cluster with open boundary conditions used for the calculation of the lower bounds.

where by $\mathscr{H}_{12345678}^{\mathrm{CG}}$ we denote the Heisenberg Hamiltonian on a complete graph spanned by the sites 1 to 8 , i.e.

$$
\mathscr{H}_{12345678}^{\mathrm{CG}}=\sum_{i=1}^{7} \sum_{j=i+1}^{8} \mathbf{S}_{i} \mathbf{S}_{j}=\frac{1}{2}\left(\sum_{i=1}^{8} \mathbf{S}_{i}\right)^{2}-\frac{1}{2} \sum_{i=1}^{8} \mathbf{S}_{i}^{2} .
$$

and similarly for the others. The nice feature of the Hamiltonian (42) is a particular hierarchy of the terms: starting from the basic building blocks - the states on sites $(1,2),(3,4)$, $(5,6)$, and $(7,8)$, chosen either as singlet or triplet - we can construct successively the definite spin states on sites $1,2,3$, and $4\left(S_{1234}\right)$ and $5,6,7$, and $8\left(S_{5678}\right)$, and finally on the whole cluster $\left(S_{12345678}\right)$, and the energy for that state can readily be determined. So the problem is reduced to bookkeeping. The spectrum is shown in Table 6

¿From the knowledge of the spectrum and degeneracies, we can calculate the exact partition function of the 8-site cluster, and from that its heat capacity and magnetic susceptibility, just as for the 4-site cluster. Expanding the magnetic susceptibility obtained in this way at high temperatures, we again find that we reproduce correctly only the first two terms of the high temperature series expansion. However empirically, the magnetic susceptibility of the 8-site cluster provides an excellent fit to experiment. The estimate of heat capacity obtained in this way is also more reliable than that found from the 4-site cluster of Section $\mathrm{A}$

\section{Lower-bound calculation}

A lower-bound estimate of the ground-state energy for spin systems can be obtained by exactly diagonalizing the Hamiltonian on a small cluster with open boundary conditions. The small cluster is chosen such that it can be used to tile the complete lattice, in which case we can write the Hamiltonian of the system as

$$
\mathscr{H}=\sum_{i} \mathscr{H}_{i}
$$


where $\mathscr{H}_{i}$ is the Hamiltonian for an individual cluster. The simplest such cluster for the square lattice with nearest neighbour bonds is the cross-linked square (tetrahedron), discussed above. The ground state wave function of the complete lattice

$$
\mathscr{H}\left|\psi_{0}\right\rangle=E_{0}\left|\psi_{0}\right\rangle
$$

can then be used as a variational wave function, providing an upper bound on the exact ground state energy $E_{\text {cluster }}$ of a single cluster

$$
\left\langle\psi_{0}\left|\mathscr{H}_{i}\right| \psi_{0}\right\rangle \geq E_{\text {cluster }}
$$

It follows that $E_{\text {cluster }}$ must in turn provide a lower bound on the ground state energy $E_{0}$ per spin of the complete lattice. This approach was introduced by Anderson [35], and later improved by Nishimori and Ozeki, who optimized the lower bound by treating the exchange on the bonds within the cluster as a variational parameter [36]. We have refined the method further by allowing additional longer range bonds in the cluster which cancel when the lattice is tiled by the sum over translated clusters. The 9-site cluster we used is shown in Fig. 26 with constraints $4 K_{1}+2 K_{1}^{\prime}=J_{1}, 2 K_{2}+2 K_{2}^{\prime}=J_{2}$ and $2 K_{3}+K_{3}^{\prime}=0$. 